\title{
Geometrical variables with direct thermodynamic significance in Lanczos-Lovelock gravity
}

\author{
Sumanta Chakraborty * and T. Padmanabhan ${ }^{\dagger}$ \\ IUCAA, Post Bag 4, Ganeshkhind, Pune University Campus, Pune 411 007, India
}

(Dated: August 9, 2018)

\begin{abstract}
It has been shown in an earlier work [arXiv:1303.1535] that there exists a pair of canonically conjugate variables $\left(f^{a b}, N_{b c}^{a}\right)$ in general relativity which also act as thermodynamically conjugate variables on any horizon. In particular their variations $\left(f^{b c} \delta N_{b c}^{a}, N_{b c}^{a} \delta f^{b c}\right)$, which occur in the surface term of the Einstein-Hilbert action, when integrated over a null surface, have direct correspondence with $(S \delta T, T \delta S)$ where $(T, S)$ are the temperature and entropy. We generalize these results to Lanczos-Lovelock models in this paper. We identify two such variables in Lanczos-Lovelock models such that (a) our results reduce to that of general relativity in the appropriate limit and (b) the variation of the surface term in the action, when evaluated on a null surface, has direct thermodynamic interpretation as in the case of general relativity. The variations again correspond to $S \delta T$ and $T \delta S$ where $S$ is now the appropriate Wald entropy for the Lanczos-Lovelock model. The implications are discussed.
\end{abstract}

\section{INTRODUCTION}

Judicious use of the principle of equivalence and principle of general covariance leads to the conclusion that gravity can be described as arising from the curvature of spacetime. These principles also go a long way in determining the kinematics of gravity viz. how spacetime curvature affects the dynamics of matter fields.

The next step is to determine the dynamics of spacetime, viz. how the matter fields act as a source for curvature. Unfortunately, there is no simple guiding principle to help us in this task. One possibility is to postulate that the field equations, derived from a suitable action principle, should not contain derivatives higher than second order. The most general action principle which will lead to this result is the LanczosLovelock action in $\mathrm{D}$ dimensions [1-4]. (In $\mathrm{D}=4$, this reduces to standard Einstein-Hilbert action.) This has been the conventional way of describing theories of gravity.

In recent years, investigations have revealed several intriguing features for this class of Lanczos-Lovelock models. They stem from the deep connection between gravitational dynamics and horizon thermodynamics, which we now know transcends general relativity. These results first emerged in the context of general relativity from the seminal work by Bekenstein, Hawking, Davies, Unruh and others showing that horizons in general (and black holes in particular) possess thermodynamic attributes like entropy [5, 6] and temperature [7-10]. Since any suitable null surface can act as a local Rindler horizon for a class of observers, this allows one to introduce observer-dependent thermodynamic variables around any event in spacetime. Then it seems natural to think of spacetime as some kind of fluid with its thermodynamic properties arising from the dynamics of underlying "atoms of spacetime." This emergent gravity paradigm (see, for a review [11-13]) has received significant amount of support from later investigations, especially from the following results:

- The gravitational field equations reduce to simple thermodynamic identities on horizons for a wide class of gravity theories more general than Einstein gravity [14-19]. The action functional for gravity can be expressed as a sum of a bulk term and a surface term with a "holographic" relation between them. Again this result holds not only in Einstein gravity but also in a more general class of theories [3, 20-22]. In fact, the action in all Lanczos-Lovelock models can be given a thermodynamic interpretation [22].

- Gravitational field equations in all the Lanczos-Lovelock models can be derived from a thermodynamic extremum principle $[23,24]$ and the action functional itself can be given a thermodynamic interpretation $[25,26]$.

\footnotetext{
* sumanta@iucaa.ernet.in; sumantac.physics@gmail.com

† paddy@iucaa.ernet.in
} 
- Gravitational field equation reduces to the Navier-Stokes equation of fluid dynamics in any spacetime, when projected on an arbitrary null surface, thereby generalizing previous results for black hole spacetime [27-29].

- More recently [30] these ideas have been taken significantly further in the context of general relativity and these results have also been generalized to Lanczos-Lovelock models [31]. It has been demonstrated that the following results hold in all these theories: (a) The total Noether charge in a 3 -volume $\mathcal{R}$, related to the time evolution vector field, can be interpreted as the heat content of the boundary $\partial \mathcal{R}$ of the volume. This provides yet another holographic result connecting the bulk and boundary variables. (b) The time evolution of the spacetime itself can be described in an elegant manner as being driven by the departure from holographic equipartition, measured by $\left(N_{\text {bulk }}-N_{\text {sur }}\right)$. The metric will be time independent in the chosen foliation if $N_{\text {sur }}=N_{\text {bulk }}$ which happens for all static geometries.

We thus have two possible routes towards gravitational dynamics. One is the conventional route, using the action functional and geometrical variables. The other is the thermodynamic route which uses suitably defined degrees of freedom, heat content etc. The link between these two routes, obviously, is provided by the action functional which - as is known from previous investigations [20,22, 25-28, 32] - has both dynamical and thermodynamical interpretation. This is a peculiar aspect of gravitational action functionals, not shared by other theories which possess no thermodynamic or emergent interpretation and hence is worth probing deeply.

A first step in this direction was taken in Ref. [33]. This work introduced two canonically conjugate variables

$$
f^{a b}=\sqrt{-g} g^{a b} ; \quad N_{a b}^{c}=Q_{a q}^{c p} \Gamma_{b p}^{q}+Q_{b q}^{c p} \Gamma_{a p}^{q} ; \quad Q_{c d}^{a b}=\frac{1}{2}\left(\delta_{c}^{a} \delta_{d}^{b}-\delta_{d}^{a} \delta_{c}^{b}\right)
$$

in the context of general relativity and showed that (the variation of) these quantities also possess simple thermodynamical interpretation in terms of $S \delta T$ and $T \delta S$. But since the thermodynamic interpretation transcends general relativity, we should be able to find similar geometrical variables in all LanczosLovelock models. The main purpose of this paper is to identify one set of such variables which satisfy the conditions: (a) These variables reduce to the ones used in general relativity in $D=4$ when the Lanczos-Lovelock model reduces to general relativity. (b) The variation of these quantities corresponds to $S \delta T$ and $T \delta S$ where $S$ is now the correct Wald entropy of the Lanczos-Lovelock model. We shall show that this can indeed be done and will discuss several properties of these variables.

The paper is organized as follows: In Sec. II we first review various properties of the Lanczos-Lovelock theories of gravity which we need for the rest of the paper. In the next section III we summarize some of the results regarding canonically conjugate variables in Einstein-Hilbert action and their possible generalization to Lanczos-Lovelock gravity. In Sec. IV we present construction of the line element near an arbitrary null surface and the thermodynamic interpretation for conjugate variables related to EinsteinHilbert action. Section V presents thermodynamic quantities for a general static spacetime and then it is generalized to arbitrary null surface constructed in the previous section for Lanczos-Lovelock gravity. The next section VI describes gravity in terms of the newly introduced conjugate variables in both EinsteinHilbert and Lanczos-Lovelock actions. Finally, we conclude with a discussion on our results. We have also presented some more details of the main calculations in two Appendices, A and B.

The metric is assumed to have signature $(-,+,+, \ldots,+)$. The fundamental constants, $G, \hbar$ and $c$ are set to unity. The latin indices $a, b, \ldots$ run over all the spacetime coordinates, the greek indices $\mu, \nu, \ldots$ over the $(D-1)$ spatial coordinates and the capitalized latin indices $A, B, \ldots$ run over the $(D-2)$ transverse coordinates when relevant.

\section{A BRIEF INTRODUCTION TO LANCZOS-LOVELOCK GRAVITY}

Consider a D-dimensional spacetime in which the gravity is described by an action functional:

$$
A=\int_{\mathcal{V}} d^{D} x \sqrt{-g} L\left(g^{a b}, R_{b c d}^{a}\right)
$$

The Lagrangian depends both on the curvature and the metric but not on the derivatives of the curvature. The most important quantity for our later purpose, derived from the Lagrangian, is the tensor:

$$
P^{a b c d}=\left(\frac{\partial L}{\partial R_{a b c d}}\right)_{g_{i j}}
$$


having all the algebraic symmetry properties of the curvature tensor. An analogue of the Ricci tensor in general relativity can also be constructed by the definition

$$
\mathcal{R}^{a b} \equiv P^{a i j k} R_{i j k}^{b} .
$$

This tensor is indeed symmetric; but the result is nontrivial to prove (for this result and other properties of these tensors, see [28]). The variation of the action presented in Eq. (2) leads to the result:

$$
\begin{aligned}
\delta A & =\delta \int_{\mathcal{V}} d^{D} x \sqrt{-g} L \\
& =\int_{\mathcal{V}} d^{D} x \sqrt{-g} E_{a b} \delta g^{a b}+\int_{\mathcal{V}} d^{D} x \sqrt{-g} \nabla_{j} \delta v^{j}
\end{aligned}
$$

where $E_{a b}$ is the field equation term and $\delta v^{a}$ is the boundary term. They are given by the following expressions:

$$
\begin{aligned}
E_{a b} & \equiv \frac{1}{\sqrt{-g}}\left(\frac{\partial \sqrt{-g} L}{\partial g^{a b}}\right)_{R_{a b c d}}-2 \nabla^{m} \nabla^{n} P_{a m n b} \\
& =\mathcal{R}_{a b}-\frac{1}{2} g_{a b} L-2 \nabla^{m} \nabla^{n} P_{a m n b} \\
\delta v^{j} & =2 P^{i b j d} \nabla_{b} \delta g_{d i}-2 \delta g_{d i} \nabla_{c} P^{i j c d}
\end{aligned}
$$

Since the quantity $P^{a b c d}$ involves second order derivative of the metric, the term $\nabla^{m} \nabla^{n} P_{a m n b}$ contains fourth order derivatives of the metric. Therefore, to get second order field equation, we must impose an extra condition on $P^{a b c d}$, such that

$$
\nabla_{a} P^{a b c d}=0
$$

Thus the problem of finding an action functional leading to a second order field equation reduces to finding scalar functions of curvature and metric such that Eq. (8) is satisfied. Such a scalar indeed exists and is unique; it is given $[2-4,16,20]$ by the Lanczos-Lovelock Lagrangian in D dimensions, as

$$
L=\sum_{m} c_{m} L_{m}=\sum_{m} \frac{c_{m}}{m} \frac{\partial L_{m}}{\partial R_{a b c d}} R_{a b c d}=\sum_{m} \frac{c_{m}}{m} P_{(m)}^{a b c d} R_{a b c d}
$$

This Lagrangian $L_{m}$ being a homogeneous function of $R_{a b c d}$ of order $m$ can also be written as $L_{m}=$ $Q_{(m)}^{a b c d} R_{a b c d}$, which can be used to identify $P_{(m)}^{a b c d}=m Q_{(m)}^{a b c d}$. From now on we shall work with this $m$ th order Lagrangian and henceforth shall drop the $m$ index. For this Lagrangian we have the following explicit expression for $P_{c d}^{a b}$ in terms of the curvature tensor:

$$
P_{c d}^{a b}=\frac{\partial L_{m}}{\partial R_{a b}^{c d}}=m \delta_{c d c_{2} d_{2} \ldots c_{m} d_{m}}^{a b a_{2} b_{2} \ldots a_{m} b_{m}} R_{a_{2} b_{2}}^{c_{2} d_{2}} \ldots R_{a_{m} b_{m}}^{c_{m} d_{m}} \equiv m Q_{c d}^{a b}
$$

This relation will be used extensively later. Also note that due to complete antisymmetry of the determinant tensor in a $\mathrm{D}$ dimensional space-time we have the following restriction $2 m \leq D$, otherwise the determinant tensor would vanish identically. Lanczos-Lovelock models at dimension $D=2 m$ are known as critical dimensions for a given Lanczos-Lovelock term. In these critical dimensions the variation of the action functional reduces to a pure surface term [34].

All generally covariant theories, including the Lanczos-Lovelock theories of gravity, possess diffeomorphism invariance. This implies that the invariance under an infinitesimal coordinate transformation, $x^{a} \rightarrow x^{a}+\xi^{a}(x)$ of the theory should lead to conservation of a current, usually called the Noether current. From variation of action functional we can get the Noether current having the following expression $[3,4,35,36]$ :

$$
J^{a} \equiv\left(2 E^{a b} \xi_{b}+L \xi^{a}+\delta_{\xi} v^{a}\right)
$$

In the above expression the last term, $\delta_{\xi} v^{a}$ represents the boundary term when the metric variation has the following form: $\delta g^{a b}=\nabla^{a} \xi^{b}+\nabla^{b} \xi^{a}$. From the property of the Noether current $\nabla_{a} J^{a}=0$, we can define an antisymmetric tensor referred to as Noether Potential by the condition, $J^{a}=\nabla_{b} J^{a b}$. Using Eq. (6) we can substitute for the the boundary term leading to an explicit form for both the Noether 
current and Potential. These general expressions can be found in [3]. In the context of Lanczos-Lovelock theories, where $\nabla_{a} P^{a b c d}=0$, they are given by

$$
\begin{aligned}
J^{a b} & =2 P^{a b c d} \nabla_{c} \xi_{d} \\
J^{a} & =2 P^{a b c d} \nabla_{b} \nabla_{c} \xi_{d}=2 \mathcal{R}^{a b} \xi_{b}+2 P_{k}{ }^{i j a} \mathcal{L}_{\xi} \Gamma_{i j}^{k}
\end{aligned}
$$

with $\Gamma_{b c}^{a}$ being the metric compatible connection.

The Noether current has a direct thermodynamic interpretation. To begin with one can associate a Wald entropy with horizons in all Lanczos-Lovelock models. The corresponding entropy density (which, integrated over the horizon gives the entropy) is given by [12, 37-42]

$$
s=-\frac{1}{8} \sqrt{\sigma} P^{a b c d} \mu_{a b} \mu_{c d}=\frac{1}{2} \sqrt{\sigma} P^{\alpha b d \beta} r_{\alpha} u_{b} u_{d} r_{\beta}
$$

It can be shown $[30,31]$ that the Noether charge inside a bulk region is $\mathcal{R}$ equal to the heat content of the boundary surface $\partial \mathcal{R}$. That is,

$$
\int_{\mathcal{V}} d^{D-1} x \sqrt{h} u^{a} J_{a}(\xi)=\epsilon \int_{\partial \mathcal{V}} d^{D-2} x T_{l o c} s
$$

which provides a direct thermodynamic as well as holographic interpretation of Noether charge. (Here $\epsilon= \pm 1$ is appropriately chosen such the the outward normal is in the direction of the acceleration of the observers; for details of this result see [30,31]).

\section{CONJUGATE VARIABLES IN EINSTEIN-HILBERT ACTION AND POSSIBLE GENERALIZATION TO LANCZOS-LOVELOCK GRAVITY}

In this section we start by reviewing the conjugate variable structure in Einstein-Hilbert action, which we will subsequently generalize to Lanczos-Lovelock gravity. We will first summarize the results for general relativity and then attempt a relatively simple generalization to Lanczos-Lovelock models, which, however, does not work. Taking a cue from this, we will define a modified set of variables, which — as we shall see — work correctly.

We start by reviewing the structural aspects of Einstein-Hilbert action. In four dimensions, the standard Lanczos-Lovelock criteria uniquely identify the action functional to be Einstein-Hilbert action:

$$
16 \pi A_{E H}=\int_{\mathcal{V}} d^{4} x \sqrt{-g} L_{E H}=\int_{\mathcal{V}} d^{4} x \sqrt{-g} R
$$

Motivated by our desire to generalize the results to Lanczos-Lovelock models, we will rewrite the above action in terms of the relevant $Q_{a}^{b c d}$ for this $m=1$ Lanczos-Lovelock model so that the Lagrangian density $L_{E H}$ becomes:

$$
L_{E H} \equiv Q_{a}^{b c d} R_{b c d}^{a} ; \quad Q_{a}^{b c d}=\frac{1}{2}\left(\delta_{a}^{c} g^{b d}-\delta_{a}^{d} g^{b c}\right)
$$

It is well known that the Einstein-Hilbert action is the sum of a bulk term, which is quadratic in the derivatives of the metric and a surface term which contains all the second derivatives [43]. This decomposition can be written as in terms of the variable $Q_{a}^{b c d}$ as

$$
\begin{aligned}
L_{E H} & =L_{\text {quad }}+L_{\text {sur }} \\
L_{\text {quad }} & \equiv 2 Q_{a}^{b c d} \Gamma_{d k}^{a} \Gamma_{b c}^{k} ; \quad L_{\text {sur }} \equiv \frac{2}{\sqrt{-g}} \partial_{c}\left(\sqrt{-g} Q_{a}^{b c d} \Gamma_{b d}^{a}\right) .
\end{aligned}
$$

The bulk part of the action, when varied, leads to Einstein equation. We stress [3] the following point: The equations of motion can be determined from the the variation of the bulk term alone. The solutions to the field equation (including, say, the black hole solutions) are not dependent on the surface term in any way since they are derived from the bulk term alone. Nevertheless, when the surface term is evaluated on the horizon it produces the entropy associated with the horizon. This is one of the hints for the thermodynamic interpretation arising from the action functional. 


\section{A. Conjugate variables in Einstein gravity}

We now review the use of a different set of canonically conjugate variables in Einstein-Hilbert action. This is done in detail in Ref. [30] and [33]; however for completeness, we review some of these results. The variational principle in general relativity becomes simpler if we introduce a new dynamical variable $f^{a b} \equiv \sqrt{-g} g^{a b}$ (which is a tensor density) instead of the metric $g_{a b}$. Then the Einstein-Hilbert action in terms of the new variable $f^{a b}$ reads $[30,33]$

$$
\sqrt{-g} R=\sqrt{-g} L_{q u a d}-\partial_{c}\left[f^{a b} \frac{\partial\left(\sqrt{-g} L_{q u a d}\right)}{\partial\left(\partial_{c} f^{a b}\right)}\right]=\sqrt{-g} L_{q u a d}-\partial_{c}\left(f^{a b} N_{a b}^{c}\right)
$$

where we have defined another quantity, representing the conjugate momenta to $f^{a b}$ as

$$
N_{a b}^{c}=\frac{\partial\left(\sqrt{-g} L_{q u a d}\right)}{\partial\left(\partial_{c} f^{a b}\right)}=Q_{a e}^{c d} \Gamma_{b d}^{e}+Q_{b e}^{c d} \Gamma_{a d}^{e}
$$

The usefulness of these variables is apparent from the structure of the variation of the Einstein-Hilbert action. These variables also lead to two terms, the equation of motion term and the surface term having the following decomposition:

$$
\delta(\sqrt{-g} R)=R_{a b} \delta f^{a b}+f^{a b} \delta R_{a b}=R_{a b} \delta f^{a b}-\partial_{c}\left(f^{a b} \delta N_{a b}^{c}\right) .
$$

Making the surface term vanishing is equivalent to demanding $\delta N_{b c}^{a}=0$, which is equivalent to setting variation of momentum to zero at the end points. (That is, we interpret Einstein-Hilbert action as a momentum space action; see p.291 of ref. [3]) Also many other expressions simplifies considerably in terms of these variables $[30,33]$. We summarize some of these important relations in terms of these variables below:

$$
\begin{aligned}
R_{a b} & =\left(-\partial_{c} N_{a b}^{c}-N_{a d}^{c} N_{b c}^{d}+\frac{1}{3} N_{a c}^{c} N_{b d}^{d}\right) \\
\sqrt{-g} R & =-f^{a b} \partial_{c} N_{a b}^{c}-\frac{1}{2} N_{a b}^{c} \partial_{c} f^{a b} \\
& =\frac{1}{2} N_{a b}^{c} \partial_{c} f^{a b}-\partial_{c}\left(f^{a b} N_{a b}^{c}\right) \\
& =-\frac{1}{2}\left[f^{a b} \partial_{c} N_{a b}^{c}+\partial_{c}\left(f^{a b} N_{a b}^{c}\right)\right] \\
\sqrt{-g} L_{\text {quad }} & =\frac{1}{2} N_{a b}^{c} \partial_{c} f^{a b} ; \quad \sqrt{-g} L_{\text {sur }}=-\partial_{c}\left(f^{a b} N_{a b}^{c}\right)
\end{aligned}
$$

The Noether current also has a simple expression [30, 33] in terms of these variables:

$$
\sqrt{-g} J^{a}=2 \sqrt{-g} R^{a b} \xi_{b}+f^{p q} \mathcal{L}_{\xi} N_{p q}^{a}
$$

Next we will consider the question of finding similar variables for Lanczos-Lovelock models.

\section{B. Conjugate variables in Lanczos-Lovelock theories}

In Einstein gravity, we have obtained a simpler description using the two conjugate variables, $f^{a b}=$ $\sqrt{-g} g^{a b}$ and $N_{a b}^{c}=Q_{a e}^{c d} \Gamma_{b d}^{e}+Q_{b e}^{c d} \Gamma_{a d}^{e}$. The most natural choice for the corresponding variables in LanczosLovelock gravity will be the ones obtained by defining $N_{b c}^{a}$ by the same relation, viz. by Eq. (20), as in general relativity but with $Q_{c d}^{a b}$ given by that for the appropriate Lanczos-Lovelock model, viz. by Eq. (10). We will first explore this choice of variable and see whether they satisfy all the requirements.

Even in Lanczos-Lovelock theories, the decomposition of the Lagrangian into a bulk term and a surface term exists and has the identical expression as in Eq. (18) with the $Q_{c d}^{a b}$ defined by Eq. (10). There are two desirable features we expect the variables to satisfy: First, the surface term should be expressible as, $-\partial(q p)$; second the quadratic part of the Lagrangian must be expressible as, $p \partial q$. Note that $q$ and $p$ are not absolute, since in the Hamiltonian formulation both are given equal weightage,and we can even interchange $q$ and $p$. In Einstein-Hilbert action these relations are given by Eq. (26).

With this motivation, let us examine whether these two variables, $\tilde{f}^{a b}$ which is same as the $f^{a b}$ in Einstein-Hilbert action and $\tilde{N}_{b c}^{a}$ having the same expression as that of $N_{b c}^{a}$ with $Q_{c d}^{a b}$ corresponding to 
the one for the Lanczos-Lovelock model, satisfy our criteria. For that purpose we need to evaluate the following combinations, $\tilde{f}^{a b} \partial_{c} \tilde{N}_{a b}^{c}$ and $\tilde{N}_{b c}^{a} \partial_{a} \tilde{f}^{b c}$. These are evaluated explicitly in Appendix A [see Eq. (A5) and Eq. (A6)]. We state here the final results:

$$
\begin{aligned}
\sqrt{-g} L_{s u r} & =-\partial_{c}\left(\tilde{f}^{p q} \tilde{N}_{p q}^{c}\right) \\
\sqrt{-g} L_{q u a d} & =\tilde{N}_{a b}^{c} \partial_{c} \tilde{f}^{a b}-\left(2 \sqrt{-g} Q_{p}^{b q c} \Gamma_{q b}^{p} \Gamma_{c m}^{m}-2 \sqrt{-g} g^{b m} Q_{a p}^{c q} \Gamma_{q b}^{p} \Gamma_{c m}^{a}\right) \\
\sqrt{-g} Q_{p}^{q r s} R_{q r s}^{p} & =-\tilde{f}^{a b} \partial_{c} \tilde{N}_{a b}^{c}-\left(2 \sqrt{-g} Q_{p}^{b q c} \Gamma_{q b}^{p} \Gamma_{c m}^{m}-2 \sqrt{-g} g^{b m} Q_{a p}^{c q} \Gamma_{q b}^{p} \Gamma_{c m}^{a}\right)
\end{aligned}
$$

Thus even though it leads to the proper surface term it does not simplify the other terms unlike in the Einstein-Hilbert case presented in Eq. (26). Hence we conclude that these variables are not suitable.

The same conclusion can also be reached from the expression for the Noether current as well. In Lanczos-Lovelock theories the Noether current has the expression given by Eq. (13), which can actually be derived without any notion of diffeomorphism invariance, using only differential geometry [30, 31]. As in the Einstein-Hilbert scenario where the last term becomes, $f^{a b} \mathcal{L}_{\xi} N_{a b}^{c}$, here also we would like the last term to be of the form $\tilde{f}^{p q} \mathcal{L}_{\xi} \tilde{N}_{p q}^{a}$. However this does not yield the correct Noether current. An extra term, depending on Lie variation of the entropy tensor $P^{a b c d}$, comes into the picture. We have used several identities regarding Lie variation of $P^{a b c d}$ but none of these help to simplify the extra term. [Though these identities do not help, they are quiet interesting and have not been derived earlier; hence we present these relations in Appendix. B]. In summary, these variables, though they are the simplest choice, do not fulfill the criteria we would like them to satisfy.

It is, however, possible to attack the problem from a different angle and obtain another set of variables that satisfies all our criteria. The clue comes from the Noether current itself. We know from Eq. (27) that in general relativity, the second term of the Noether current, given by $f^{a b} \mathcal{L}_{\xi} N_{a b}^{c}$, has the two variables $\left[f^{a b}, N_{i j}^{c}\right]$ on the two sides of $\mathcal{L}_{\xi}$ while in the $m$ th order Lanczos-Lovelock model the corresponding term $P_{k}^{a j i} \mathcal{L}_{\xi} \Gamma_{a j}^{k}=m Q_{k}^{a j i} \mathcal{L}_{\xi} \Gamma_{a j}^{k}$ (see Eq. (13)) has the variables $\left[\Gamma_{a j}^{k}, Q_{k}{ }^{a j i}\right]$ on the two sides of $\mathcal{L}_{\xi}$. Taking a cue from this, let us define two variables:

$$
\begin{aligned}
\Gamma_{b c}^{a} & =\frac{1}{2} g^{a d}\left(-\partial_{d} g_{b c}+\partial_{b} g_{c d}+\partial_{c} g_{b d}\right) \\
U_{a}^{b c d} & =2 \sqrt{-g} Q_{a}^{b c d}
\end{aligned}
$$

where $\Gamma_{b c}^{a}$, of course, is the standard connection and $Q_{a}^{b c d}$ is given by Eq. (10). (The factors are chosen to give the correct limit for general relativity when $m=1$; we will see that these definitions work.)

Interestingly, these variables can be introduced in a somewhat different manner as well. Suppose we consider the Lanczos-Lovelock model Lagrangian which can be expressed entirely in terms of $R_{k l}^{i m}$ [with index placements $(2,2)]$ plus Kronecker deltas. Since $R_{k l}^{i m}=g^{m j} R_{j k l}^{i}$ it can also be expressed in terms of the variables $R_{j k l}^{i}$ [with index placements $\left.(1,3)\right]$ and the metric $g^{a b}$. On the other hand, $R_{j k l}^{i}[$ with index placements $(1,3)]$ can be written entirely in terms of $\partial_{l} \Gamma_{j k}^{i}$ and $\Gamma_{j k}^{i}$ without the metric appearing anywhere. Therefore, one can think of the Lanczos-Lovelock Lagrangian, when expressed in terms of $R^{i}{ }_{j k l}$ [with index placements $\left.(1,3)\right]$ and the metric $g^{a b}$ as a functional of $\left[g^{a b}, \partial_{l} \Gamma_{j k}^{i}, \Gamma_{j k}^{i}\right]$. This suggests defining the "conjugate variable" to the connection:

$$
m U_{u}{ }^{v l w} \equiv \frac{\partial(\sqrt{-g} L)}{\partial\left(\partial_{l} \Gamma_{v w}^{u}\right)}
$$

The equality of the two sides, for $m$-th order Lanczos-Lovelock Lagrangian is easy to verify using Eq. (A9). It can be proved that this quantity $U_{a b c d}$ has all the symmetries of the curvature tensor (essentially because the $\partial_{l} \Gamma_{v w}^{u}$ occurs in $L$ only through the curvature tensor.) This suggests yet another meaning to the variables introduced in Eqs. (31) and (32).

Of course, this set of variables in Eqs. (31) and (32) works for the Einstein-Hilbert case well. Now $U_{u}{ }^{v l w}$ can be expressed entirely in terms of the metric and - in this sense - one can think of the metric (or rather the particular combination of metric components) as conjugate to connection (rather than the other way around!) even in the Einstein-Hilbert case. All the original relations for the Einstein-Hilbert action can be written in terms of these two variables instead of $f^{a b}$ and $N_{b c}^{a}$ as in Eqs. (A7) and (A8) matching exactly Eq. (26). The reason has to do with the fact that for any covariant derivative operator 
$\hat{D}$ we have

$$
\begin{aligned}
f^{p q} \hat{D} N_{p q}^{a} & =2 \sqrt{-g} g^{p q} \hat{D}\left(Q_{p d}^{a e} \Gamma_{e q}^{d}\right) \\
& =2 \sqrt{-g} g^{p q} Q_{p d}^{a e} \hat{D} \Gamma_{e q}^{d} \\
& =2 \sqrt{-g} Q_{d}^{q e a} \hat{D} \Gamma_{q e}^{d}=U_{p}^{q r a} \hat{D} \Gamma_{q r}^{p}
\end{aligned}
$$

This is possible only in the Einstein-Hilbert action where $Q_{a b}^{c d}$ involves only the Kronecker delta functions and hence can be pulled through any covariant derivative operator. Thus, in general, the pair $\left[\Gamma_{b c}^{a}, U_{a}^{p q r}\right]$ can also act as the conjugate variables, in Einstein gravity. Since $U_{a}{ }^{p q r}$ can be entirely expressed in terms of the metric in general relativity, they are equivalent to $f^{a b}$ and $N_{a b}^{c}$ structurally. However in higher order Lanczos-Lovelock theories $Q_{c d}^{a b}$ depends on curvature tensor and hence the above equivalence is broken. As we shall see it is better to work with the two variables as in Eqs. (31) and (32), as shown in App. A Eqs. (A7) and (A8).

Finally, note that, by construction, the Noether current becomes in terms of these variables

$$
\begin{aligned}
\sqrt{-g} J^{a} & =m\left(2 U_{b}{ }^{p q r} R^{a}{ }_{p q r} v^{b}+U_{b}{ }^{c d a} \mathcal{L}_{\xi} \Gamma_{c d}^{b}\right) \\
& =2 \sqrt{-g} \mathcal{R}_{b}^{a} v^{b}+m U_{b}{ }^{c d a} \mathcal{L}_{\xi} \Gamma_{c d}^{b}
\end{aligned}
$$

This is exactly of the same form as Eq. (27), the Noether current for Einstein-Hilbert action.

\section{THERMODYNAMIC STRUCTURE OF EINSTEIN-HILBERT ACTION}

In this section we will rapidly review the thermodynamic structure of conjugate variables introduced in an earlier work [33] and then shall extend these thermodynamic variables to Lanczos-Lovelock gravity. For the geometrical construction, we shall confine ourselves in a four-dimensional spacetime, with the possibility of generalizing to higher dimensions in a straightforward manner. We start by reviewing the construction of metric near an arbitrary null surface.

\section{A. Construction of Gaussian null coordinates}

Let us consider the four-dimensional spacetime $V^{4}=M^{3} \times R$, where $M^{3}$ is a compact three-dimensional manifold. We will consider spacetimes to be time orientable with null embedded hypersurfaces, which are diffeomorphic to $M^{3}$ with closed null generators. We take $\mathcal{N}$ to be such a null hypersurface with null generator $[44,45]$. On this null surface $\mathcal{N}$ we can introduce spacelike two surface with coordinates $\left(x_{1}, x_{2}\right)$ defined on them. The null geodesics generating the null hypersurface $\mathcal{N}$ goes out of this spacelike two surface. Thus we can use these null generators to define coordinates on the null hypersurface. The intersection point of these null geodesics with the spacelike two surface can be determined by the coordinates $\left(x_{1}, x_{2}\right)$, which then evolves along the null geodesic, which is affinely parametrized by $u$, and label each point on the null hypersurface as $\left(u, x_{1}, x_{2}\right)$. The above system of coordinates readily identify three basis vectors: (a) the tangent to the null geodesics, $\ell=\partial / \partial u$, and (b) basis vectors on the two surface $\mathbf{e}_{A}=\partial / \partial_{A}$.

Having fixed the coordinates on the null surface $\mathcal{N}$ we now move out of the surface using another set of null generators with tangent $k^{a}$ satisfying the following constraints: (a) $k_{a} k^{a}=0$, (b) $e_{A}^{a} k_{a}=0$ and finally $\ell_{a} k^{a}=-1$. These null geodesics are taken to intersect the null surface at coordinates $\left(u, x_{1}, x_{2}\right)$ and then move out with affine parameter $r$, such that any point in the neighborhood of the null surface can be characterized by four coordinates $\left(u, r, x_{1}, x_{2}\right)$. In this coordinate system, the null surface is given by the condition $r=0$. This defines a coordinate system $\left\{u, r, x^{A}\right\}$ over the global manifold $V^{4}$. This system of coordinates are formed in a manner analogous with Gaussian Normal Coordinate and hence is referred to as Gaussian null coordinates $(G N C)$.

Having set the full coordinate map near the null surface we now proceed to determine the metric elements in that region. Note that $\ell_{a} \ell^{a}=0$ leads to $g_{u u}=0$ on the null surface since $\ell=\partial / \partial u$. We also note that the basis vectors $e_{A}^{a}$ have to lie on the null surface implying $\ell_{a} e_{A}^{a}=0$ on $\mathcal{N}$, which leads to $g_{u A}=0$. Also the metric on the two-surface is given by $g_{A B}=e_{A}^{a} e_{B}^{b} g_{a b}$, which we denote by $\mu_{A B}$. We also need the criteria that $\mu_{A B}$ is positive definite with finite determinant ensuring invertibility and non-degeneracy of the two metric. Thus the following metric components gets fixed to be:

$$
\begin{array}{r}
\left.g_{u u}\right|_{r=0}=\left.g_{u A}\right|_{r=0}=0 ; \\
g_{A B}=\mu_{A B}
\end{array}
$$


Let us now proceed to determine the other components of the metric. For this, we will use the vector $\mathbf{k}=-\partial / \partial r$ such that from $k^{a} k_{a}=0$ we get $g_{r r}=0$ throughout the spacetime manifold. Also from the criteria that the null geodesics are affinely parametrized by $r$ we readily obtain $\partial_{r} g_{r \alpha}=0$, where $\alpha=\left(u, x_{1}, x_{2}\right)$. Again, from the conditions $\ell^{a} k_{a}=-1$, we readily get $g_{r u}=1$ and from $k_{a} e_{A}^{a}=0$ we get $g_{r A}=0$. From the criteria derived earlier showing $\partial_{r} g_{r \alpha}=0$ we can conclude that the above two-metric coefficients are valid everywhere. Thus within the global region $V^{4}$ we can have smooth functions $\alpha$ and $\beta_{A}$ such that, $\left.\alpha\right|_{r=0}=\left.\left(\partial g_{u u} / \partial r\right)\right|_{r=0}$ and $\left.\beta_{A}\right|_{r=0}=\left.\left(\partial g_{u A} / \partial r\right)\right|_{r=0}$. With these two identifications we have the following expression for the line element as

$$
d s^{2}=g_{a b} d x^{a} d x^{b}=-2 r \alpha d u^{2}+2 d u d r-2 r \beta_{A} d u d x^{A}+\mu_{A B} d x^{A} d x^{B}
$$

where $\mu_{A B}$ is the two-dimensional metric representing the metric on the null surface. Note that the construction presented above is completely general; it can be applied in the neighborhood of any null hypersurface and, in particular, to the event horizon of a black hole.

\section{B. Thermodynamics related to Einstein-Hilbert action}

In this section we will show that the variables $f^{a b}=\sqrt{-g} g^{a b}$ and $N_{b c}^{a}=Q_{b e}^{a d} \Gamma_{c d}^{e}+Q_{c e}^{a d} \Gamma_{b d}^{e}$ are closely related to the thermodynamic properties of null surfaces. The variations $p \delta q$ and $q \delta p$ obtained from conjugate variables have direct thermodynamic interpretation associated with them. This result was obtained earlier in Ref. [33] but we go through the key steps for completeness as well as for a minor relaxation of the conditions originally used in Ref. [33].

\section{Static Spacetime}

We will first prove these relations for a general static spacetime before discussing arbitrary null surface constructed above. Let us consider an arbitrary static spacetime with horizon. For this spacetime we have an arbitrary two surface with metric $\sigma_{A B}$ and the line element can be written in the form [46]:

$$
d s^{2}=-N^{2} d t^{2}+d n^{2}+\sigma_{A B} d y^{A} d y^{B}
$$

In the above line element, $n$ represents spatial direction normal to the $(D-2)$ dimensional hypersurface with $\sigma_{A B}$ being transverse metric on the two surface. Let $\boldsymbol{\xi}=\partial / \partial t$ be a timelike Killing vector, with Killing horizon located at, $N^{2} \rightarrow 0$. The coordinate system is chosen in such a way that $n=0$ on the Killing horizon. Then, in the near horizon regime, the following expansions of $N$ and $\sigma_{A B}$ are valid [46]:

$$
\begin{aligned}
N & =\kappa\left(x^{A}\right) n+\mathcal{O}\left(n^{3}\right) \\
\sigma_{A B} & =\left[\sigma_{H}(y)\right]_{A B}+\frac{1}{2}\left[\sigma_{2}(y)\right]_{A B} n^{2}+\mathcal{O}\left(n^{3}\right)
\end{aligned}
$$

In the above expression $\kappa$ is the local gravitational acceleration defined as: $\kappa=\partial_{n} N$ and in the $r \rightarrow 0$ limit, $\kappa \rightarrow \kappa_{H}$, satisfying all the standard properties of surface gravity. Also $\kappa / N$ represents the normal component of the four acceleration of an observer at fixed $\left(n, x^{A}\right)$. Throughout the calculation we shall evaluate quantities on a $n=$ constant surface and then take the $n \rightarrow 0$ limit. The nonzero components of the metric variations are:

$$
\delta g_{t t}=2 N \delta N ; \quad \delta g_{A B}=\delta \sigma_{A B}
$$

We will also require the nonzero components of $N_{b c}^{a}$ which are [33]:

$$
N_{t t}^{n}=-N \partial_{n} N ; \quad N_{n n}^{n}=\frac{\partial_{n} N}{N}+\frac{1}{2} \sigma^{A B} \partial_{n} \sigma_{A B} ; \quad N_{A B}^{n}=\frac{1}{2} \partial_{n} \sigma_{A B}
$$

Then in the near horizon limit the respective $p \delta q$ and $q \delta p$ terms turn out to be:

$$
\left.N_{a b}^{n} \delta f^{a b}\right|_{H}=2 \kappa \delta(\sqrt{\sigma}) ; \quad f^{a b} \delta N_{a b}^{n}=2 \sqrt{\sigma} \delta \kappa
$$

Then integration over the transverse variables and introduction of proper numerical factor yields the variation of the surface term in Einstein-Hilbert action to be :

$$
\begin{aligned}
\frac{1}{16 \pi} \int d^{2} x_{\perp} N_{a b}^{n} \delta f^{a b} & =\int d^{2} x \frac{\kappa}{2 \pi} \delta\left(\frac{\sqrt{\sigma}}{4}\right)=\int d^{2} x T \delta s \\
\frac{1}{16 \pi} \int d^{2} x_{\perp} f^{a b} \delta N_{a b}^{n} & =\int d^{2} x \frac{\sqrt{\sigma}}{4} \delta\left(\frac{\kappa}{2 \pi}\right)=\int d^{2} x s \delta T
\end{aligned}
$$


where $s=\sqrt{\sigma} / 4$ is the entropy density of the spacetime. (If the surface gravity $\kappa$ is independent of the transverse coordinates then the above results become $T \delta S$ and $S \delta T$ respectively, but the result in the above form expressed through Eqs. (43) and (44) is more generally applicable.) The above equations can also be written in terms of $U_{a}^{b c d}$ and $\Gamma_{b c}^{a}$, since $f^{a b} \delta N_{a b}^{c}=2 \sqrt{-g} g^{a b} Q_{a d}^{c e} \delta \Gamma_{b e}^{d}=U_{d}^{b e c} \delta \Gamma_{b e}^{d}$ Hence Eqs. (43) and (44) can also be presented in terms of the variables $U_{a}^{b c d}$ and $\Gamma_{b c}^{a}$ as:

$$
\begin{aligned}
\delta H_{\text {sur }}^{(1)} & =\frac{1}{16 \pi} \int d^{2} x U_{a}^{b c r} \delta \Gamma_{b c}^{a}=\int d^{2} x s \delta T \\
\delta H_{\text {sur }}^{(2)} & =\frac{1}{16 \pi} \int d^{2} x \Gamma_{b c}^{a} \delta U_{a}^{b c r}=\int d^{2} x T \delta s
\end{aligned}
$$

However, as we will see later, this not possible in Lanczos-Lovelock gravity, and we have to work with $U_{d}^{\text {bec }}$ directly.

\section{Generalization to Arbitrary null surface}

In the context of horizon thermodynamics the concepts of local Rindler frame and Rindler horizons are extensively used [32], where local Rindler horizon refers to a patch of null surface which is perceived as the horizon by a suitably defined accelerated observer. This analysis can be made more general by working in the near horizon regime of an arbitrary null surface. Its construction was presented in Sec. IV A leading to the line element in the near horizon limit being given by Eq. (37), with $r=0$ representing the null surface.

It will, however, be unrealistic to expect arbitrary variations of the metric in the near horizon limit of an arbitrary null surface to have simple thermodynamic interpretation. In order to obtain suitable thermodynamic interpretation we need to impose some restrictions. These restriction can be of two types: (i) Those involving restriction on the background metric itself with variations being arbitrary. This is broadly what we did in the last section where we assumed the background to be static but kept the variations arbitrary. (ii) Those keeping background metric arbitrary with some restrictions being imposed on the variations. Among these two, restriction on the variation is conceptually preferable since we want the background metric to represent any arbitrary null surface and hence we shall keep it most general. As we shall see, the conditions on the metric variation we need to impose turn out to be $\partial_{u} \delta\left(\mu_{A B}\right)=0$ and $\partial_{u} \delta(\sqrt{\mu})=0$. These conditions imply that these specific variations should not depend on time coordinate $u$ and thus henceforth will be referred to as stationarity conditions in what follows. (These conditions also generalize slightly the results obtained earlier in [33]).

We now proceed to calculate the surface term which turns out to be:

$$
A_{\text {sur }}=-\frac{1}{16 \pi} \int d^{3} x n_{c} f^{a b} N_{a b}^{c}
$$

One of the integral in the above expression is over time which will just lead to a multiplicative factor in static geometries. It is, therefore, more convenient to work with the surface Hamiltonian (which is actually the heat density of the surface; see [30]) defined as:

$$
H_{\text {sur }}=-\frac{\partial A_{\text {sur }}}{\partial u}=\frac{1}{16 \pi} \int d^{2} x n_{c} f^{a b} N_{a b}^{c}
$$

The surface Hamiltonian in Einstein-Hilbert action, evaluated for the above metric, turns out to be [33]:

$$
\begin{aligned}
H_{\text {sur }} & =-\frac{1}{16 \pi} \int d^{2} x \sqrt{h} n_{r} V^{r} \\
& =-\frac{1}{16 \pi} \int d^{2} x\left[-\frac{1}{\sqrt{\mu}} \partial_{u} \mu-\sqrt{\mu}\left(2 \alpha+2 r \partial_{r} \alpha+2 r \beta^{2}+2 r^{2} \beta_{A} \partial_{r} \beta^{A}\right)\right. \\
& \left.-\frac{2 r \alpha+r^{2} \beta^{2}}{\sqrt{\mu}} \partial_{r} \mu-\sqrt{\mu} r \partial_{A} \beta^{A}-\frac{r \beta^{A}}{\sqrt{\mu}} \partial_{A} \mu\right] .
\end{aligned}
$$

Since integration variables are $u$ and $x^{A}$, we can take the $r \rightarrow 0$ limit easily leading to, $\sqrt{h} n_{r} V^{r}=$ $-\partial_{u} \mu / \sqrt{\mu}-2 \sqrt{\mu} \alpha$. The first term vanishes when stationarity conditions are used. We then arrive at:

$$
H_{\text {sur }}=\frac{1}{16 \pi} \int d^{2} x 2 \sqrt{\mu} \alpha=\int d^{2} x\left(\frac{\alpha}{2 \pi}\right)\left(\frac{\sqrt{\mu}}{4}\right)=\int d^{2} x T s
$$


which is indeed the heat density at the boundary.

The variation of this surface Hamiltonian splits into two parts such that:

$$
\delta H_{\text {sur }}=\frac{1}{16 \pi} \int d^{2} x n_{c}\left(f^{a b} \delta N_{a b}^{c}+N_{a b}^{c} \delta f^{a b}\right) \equiv \delta H_{\text {sur }}^{(1)}+\delta H_{\text {sur }}^{(2)}
$$

where $\delta H_{\text {sur }}^{(1)}$ contains $f^{a b} \delta N_{a b}^{c}$ and $\delta H_{\text {sur }}^{(2)}$ contains $N_{a b}^{c} \delta f^{a b}$. Among them $f^{a b} \delta N_{a b}^{c}$ has the following expression near the $r=0$ null surface:

$$
f^{a b} \delta N_{a b}^{r}=2 \sqrt{\mu} \delta\left(\alpha+\frac{1}{4} \mu^{A C} \partial_{u} \mu_{A C}\right)+\frac{1}{2} \sqrt{\mu} \mu^{A B} \partial_{u} \delta \mu_{A B}
$$

It is evident from the above expression that the stationarity conditions mentioned above leads to the following result:

$$
\delta H_{\text {sur }}^{(1)} \equiv \frac{1}{16 \pi} \int d^{2} x f^{a b} \delta N_{a b}^{r}=\frac{1}{8 \pi} \int d^{2} x \sqrt{\mu} \delta \alpha
$$

Rather than evaluating the second term $N_{a b}^{c} \delta f^{a b}$ individually it is a little simpler to obtain it by considering variation of the total surface Hamiltonian. The variation of the full surface term is:

$$
\delta H_{\text {sur }}=\frac{1}{16 \pi} \int d^{2} x \delta(2 \sqrt{\mu} \alpha) .
$$

Using this result and Eq. (53) the second variation in Eq. (51) becomes,

$$
\delta H_{\text {sur }}^{(2)}=\frac{1}{16 \pi} \int d^{2} x N_{a b}^{r} \delta f^{a b}=\frac{1}{8 \pi} \int d^{2} x \alpha \delta \sqrt{\mu}
$$

We can rewrite the integrands of Eqs. (53) and (55) as $s \delta T$ and $T \delta s$ respectively. More formally, we write:

$$
\begin{aligned}
\delta H_{\text {sur }}^{(1)} & =\frac{1}{16 \pi} \int d^{2} x f^{a b} \delta N_{a b}^{r} \\
& =\int d^{2} x \frac{\sqrt{\mu}}{4} \delta\left(\frac{\alpha}{2 \pi}\right)=\int d^{2} x s \delta T \\
\delta H_{\text {sur }}^{(2)} & =\frac{1}{16 \pi} \int d^{2} x N_{a b}^{r} \delta f^{a b} \\
& =\int d^{2} x \frac{\alpha}{2 \pi} \delta\left(\frac{\sqrt{\mu}}{4}\right)=\int d^{2} x T \delta s
\end{aligned}
$$

where again $s=\sqrt{\mu} / 4$ is the entropy density of the spacetime.

This result brings out the connection between these conjugate variables and respective thermodynamic quantities pertaining to the null surface acting as a local Rindler horizon. The curious feature is that while the surface term integrated over the horizon leads to the heat content, the variation $T \delta s$ itself comes from variation of generalized coordinates and the variation $s \delta T$ coming from variation of generalized momenta.

Finally, as in the previous section these relations can also be expressed in terms of $U_{a}^{b c d} \delta \Gamma_{b c}^{a}$ and $\Gamma_{b c}^{a} \delta U_{a}^{b c d}$ respectively. That is

$$
\begin{aligned}
\delta H_{\text {sur }}^{(1)} & =\frac{1}{16 \pi} \int d^{2} x U_{a}^{b c r} \delta \Gamma_{b c}^{a}=\int d^{2} x s \delta T \\
\delta H_{\text {sur }}^{(2)} & =\frac{1}{16 \pi} \int d^{2} x \Gamma_{b c}^{a} \delta U_{a}^{b c r}=\int d^{2} x T \delta s
\end{aligned}
$$

Next we will take up the case of Lanczos-Lovelock models.

\section{THERMODYNAMICS RELATED TO LANCZOS-LOVELOCK ACTION}

In this section, we shall describe the corresponding results for the Lanczos-Lovelock models. As in the previous case, we shall first illustrate the results for a general static spacetime and then generalize these results to the arbitrary null surface constructed in Sec. IV A. 
The surface term in Lanczos-Lovelock Lagrangian, discussed in Sec. II, can be written as:

$$
-\partial_{c}\left(2 \sqrt{-g} Q_{a}^{b d c} \Gamma_{b d}^{a}\right) \equiv-\partial_{c}\left(\sqrt{-g} V^{c}\right)
$$

Then under infinitesimal variation the surface term variation can be subdivided into two parts

$$
\delta\left(\sqrt{-g} V^{c}\right)=U_{a}^{b d c} \delta \Gamma_{b d}^{a}+\Gamma_{b d}^{a} \delta U_{a}^{b d c}
$$

where one term involves variation of connections, while the other one is quiet complex and involves variation of both the metric and the entropy tensor. Hence the variation of the surface term can be written by introducing the surface Hamiltonian in an identical manner as follows:

$$
\begin{aligned}
\delta H_{\text {sur }} & =\frac{1}{16 \pi} \int d^{D-2} x n_{c} \delta\left(\sqrt{-g} V^{c}\right) \\
& =\frac{1}{16 \pi} \int d^{D-2} x n_{c} U_{a}^{b d c} \delta \Gamma_{b d}^{a}+\frac{1}{16 \pi} \int d^{D-2} x n_{c} \Gamma_{b d}^{a} \delta U_{a}^{b d c} \\
& =\delta H_{\text {sur }}^{(1)}+\delta H_{\text {sur }}^{(2)}
\end{aligned}
$$

Also the variation of the connection due to infinitesimal change of metric $g_{a b} \rightarrow g_{a b}+h_{a b}$ is:

$$
\delta \Gamma_{q r}^{p}=\frac{1}{2} h^{p a}\left(-\partial_{a} g_{q r}+\partial_{q} g_{a r}+\partial_{r} g_{a q}\right)+\frac{1}{2} g^{p a}\left(-\partial_{a} h_{q r}+\partial_{q} h_{a r}+\partial_{r} h_{a q}\right)
$$

Using these results we can calculate the variation explicitly for different metrics. We will show that, just as in the case of Einstein-Hilbert action, the first term in Eq. (62) leads to $s \delta T$ while the second term leads to $T \delta s$.

\section{A. A general static spacetime}

We will consider an arbitrary static spacetime with horizons as presented in Sec. IV B 1. For the line element presented in Eq. (38) using Eq. (A10) and Eq. (39) we find that (in the relevant $n \rightarrow 0$ limit) only three components of connection are non zero. These are : $\Gamma_{t t}^{n}=N \partial_{n} N, \Gamma_{n t}^{t}=\partial_{n} N / N$ and $\Gamma_{B C}^{A}$. Another two expressions we need for the calculation are the $(D-2)$ dimensional surface element and Wald entropy for Lanczos-Lovelock theories. The null surface we are interested in can be defined by the condition, $l^{2}=0$, with $l_{a}$ being (local) Killing vector. Then if we can introduce another auxiliary null vector $k_{a}$ such that, $l_{a} k^{a}=-1$, then the $(D-2)$ dimensional surface element turns out to be:

$$
d \Sigma_{a b}=d^{D-2} x \mu_{a b}=-d^{D-2} x\left(l_{a} k_{b}-l_{b} k_{a}\right) .
$$

The Wald entropy expressed in terms of the entropy tensor is [38, 47]:

$$
S=-\frac{1}{8} \int \sqrt{\sigma} d^{D-2} x P^{a b c d} \mu_{a b} \mu_{c d}
$$

where $\sigma$ is the determinant of the metric on the two surface. With the $l_{a}$ and $k_{a}$ defining the bi-normal of the $(D-2)$ dimensional surface, we have the following expression for the entropy of this static horizon:

$$
S=\frac{1}{2} \int d^{D-2} x \sqrt{\sigma} P_{n t}^{n t} \equiv \int d^{D-2} x s
$$

where $s=(1 / 2) \sqrt{\sigma} P_{n t}^{n t}$. Next we evaluate the variation $U_{a}^{b d n} \delta \Gamma_{b d}^{a}$ which turns out to be:

$$
\begin{aligned}
\left.U_{a}^{b d n} \delta \Gamma_{b d}^{a}\right|_{H} & =2 \sqrt{-g} Q_{a}^{b d n} \delta \Gamma_{b d}^{a} \\
& =2 N^{2} \sqrt{\sigma} Q_{n}^{t t n} \delta\left(\partial_{n} N\right)+2 \sqrt{\sigma} Q_{t}{ }^{n t n} \delta\left(\partial_{n} N\right) \\
& =4 \sqrt{\sigma} Q_{n t}^{n t} \delta \kappa
\end{aligned}
$$

Along the similar lines we can compute the other part of the variation, $\Gamma_{b d}^{a} \delta U_{a}^{b d n}$ which can be written as:

$$
\left.\Gamma_{b d}^{a} \delta U_{a}^{b d n}\right|_{H}=4 \kappa \delta\left(\sqrt{\sigma} Q_{n t}^{n t}\right)
$$


From Eq. (66) we get the expression for entropy and the temperature is given by as $\kappa / 2 \pi$. With these two identifications we find:

$$
\begin{aligned}
\frac{1}{16 \pi} \int d^{D-2} x \Gamma_{b d}^{a} \delta\left(m U_{a}^{b d n}\right) & =\frac{1}{2} \int d^{D-2} x \frac{\kappa}{2 \pi} \delta\left(\sqrt{\sigma} P_{n t}^{n t}\right)=\int d^{D-2} x T \delta s \\
\frac{1}{16 \pi} \int d^{D-2} x\left(m U_{a}^{b d n}\right) \delta \Gamma_{b d}^{a} & =\frac{1}{2} \int d^{D-2} x \sqrt{\sigma} P_{n t}^{n t} \delta\left(\frac{\kappa}{2 \pi}\right)=\int d^{D-2} x s \delta T
\end{aligned}
$$

In the above expressions $s$ represents the entropy density of the horizon, which reduces to $\sqrt{\sigma} / 4$ in the Einstein-Hilbert limit. The above results show that the two terms in Eq. (62) leads to $T \delta s$ and $s \delta T$ respectively, with $\kappa$ being $\kappa\left(x^{A}\right)$. Also we observe that these are identical to those obtained in EinsteinHilbert scenario as presented in Eqs. (45) and (46) respectively. (As in the general relativity, if $\kappa$ is independent of transverse coordinates $[48,49]$ one can integrate to obtain $T \delta S$ and $S \delta T$ respectively; but these results are more general.)

Thus we have natural generalization of the results in general relativity to all Lanczos-Lovelock models if we use the variables $U_{a}^{b c d}$ and $\Gamma_{b c}^{a}$ introduced in Sec. III.

\section{B. Generalization to arbitrary null surface}

Having discussed the thermodynamic interpretation of the two variables for a general static spacetime, we will now extend the result to an arbitrary null surface. The metric near the null surface has been constructed in Sec. IV A and we shall use that metric to evaluate various quantities of interest. As in the case of static situation, here also we shall calculate all the quantities on a $r=$ constant surface and then shall take the limit $r \rightarrow 0$ to retrieve the null surface. For that purpose we start with normal to the $r=$ constant surface and then take the null limit. With proper choice of $l_{a}$ and $k_{a}$ [50] the entropy turns out to be:

$$
S=\frac{1}{2} \int d^{D-2} x \sqrt{\mu} P_{u r}^{u r}
$$

Let us next calculate the surface Hamiltonian that comes from the $r=$ constant surface. Surface Hamiltonian has the following expression

$$
H_{\text {sur }}=\frac{1}{8 \pi} \int d^{D-2} x \sqrt{\mu} Q_{a}^{b d r} \Gamma_{b d}^{a}
$$

The only connection components that will remain nonzero in the $r \rightarrow 0$ limit are those given by Eq. (A11). With these connections the surface Hamiltonian turns out to be,

$$
\begin{aligned}
H_{\text {sur }} & =\frac{1}{8 \pi} \int d^{D-2} x \sqrt{\mu}\left[2 \alpha Q_{u r}^{u r}+2 \beta_{A} Q_{u r}^{A r}+\mu^{B D} \hat{\Gamma}_{B C}^{A} Q_{A D}^{C r}-\mu^{A C} \partial_{r} \mu_{A B} Q_{u C}^{B r}\right. \\
& \left.+\frac{1}{2}\left\{-\partial_{u} \mu_{A B} Q_{r}^{A B r}+\partial_{u} \mu_{B C}\left(Q^{C B u r}+Q^{C u B r}\right)\right\}\right]
\end{aligned}
$$

In the Einstein-Hilbert limit only the $Q_{u r}^{u r}$ term contributes leading to Eq. (53). Now using Eq. (10) all these $Q_{c d}^{a b}$ terms can be calculated. They lead to:

$$
\begin{aligned}
& Q_{u r}^{A r}=\delta_{u r C D R S \ldots}^{A r u B P Q \cdots} R_{u B}^{C D} R_{P Q}^{R S} \ldots \\
& Q_{A D}^{C r}=\delta_{A D u r P Q \cdots}^{C r M N u L} R_{M N}^{u r} R_{u L}^{P Q} \ldots+\delta_{A D u r R S \ldots}^{C r u M P Q \ldots} R_{u M}^{u r} R_{P Q}^{R S} \ldots \\
& +\delta_{A D u Q L J r W \ldots}^{C r M P u K U V} R_{M P}^{u Q} R_{u K}^{L J} R_{U V}^{r W} \ldots+\delta_{A D u Q r K X Y \ldots}^{C r M P u L U V \ldots} R_{M P}^{u Q} R_{u L}^{r K} R_{U V}^{X Y} \ldots \\
& +\delta_{A D u Q r L X Y \cdots}^{C r u P M N U V} R_{u P}^{u Q} R_{M N}^{r L} R_{U V}^{X Y} \cdots \\
& Q_{u C}^{B r}=\delta_{u C r D \ldots}^{B r u A \ldots} R_{u A}^{r D} \ldots+\delta_{u C Q R r M \ldots}^{B r u P J K \ldots} R_{u P}^{Q R} R_{J K}^{r M} \ldots \\
& Q_{r A}^{B r}=\delta_{r A u D P Q \ldots}^{B r u C M N \cdots} R_{u C}^{u D} R_{M N}^{P Q} \ldots+\delta_{r A E F u R \ldots}^{B r u C M N \cdots} R_{u C}^{E F} R_{M N}^{u R} \cdots \\
& Q_{r u}^{C B}=\delta_{r u P Q R S \ldots}^{C B r u M N} R_{r u}^{P Q} R_{M N}^{R S} \ldots+\delta_{r u Q R N S \ldots}^{C B r P u M \ldots} R_{r P}^{M N} R_{u M}^{N S} \ldots
\end{aligned}
$$

In arriving at the above results we have used the fact that the determinant tensor is antisymmetric in any two indices. Thus all the remaining terms, in the above expressions, contain only the components 
of the curvature tensor with indices depending on coordinates on the null surface. They are all fully characterized by the $(D-2)$ metric $\mu_{A B}$. In the null limit we have:

$$
R_{u B}^{C Q}=\mu^{Q P} R_{P u B}^{C} ; \quad R_{J K}^{r M}=\mu^{M N} R_{N J K}^{r} ; \quad R_{u C}^{r D}=-\mu^{A D} R_{A C u}^{r} ;
$$

Having obtained all the components of the surface term we will now consider the thermodynamic interpretation.

As we said earlier thermodynamic interpretation can be given provided some additional conditions are imposed. As explained in Sec. IV B 2 we can use two type of conditions: either on the variation (with the metric remaining arbitrary) or on the metric (keeping the variations arbitrary). As in the case of general relativity, it is preferable to impose the conditions the variations but keeping the background metric arbitrary. From the result we had in the Einstein-Hilbert action we would expect these conditions to include $\delta\left(\mu^{C A} \partial_{i} \mu_{A B}\right)=0, \hat{D}_{M} \delta \alpha=0$ and $\partial_{i} \delta \mu_{A B}=0$. It turns out that, along with these conditions we also need to set $\delta \beta_{A}=0$. With these conditions we get the only non-zero contributing term as:

$$
H_{\text {sur }}=\frac{1}{4 \pi} \int d^{D-2} x \sqrt{\mu} \alpha Q_{u r}^{u r}=\frac{1}{m} \int d^{D-2} x\left(\frac{\alpha}{2 \pi}\right)\left(\frac{1}{2} \sqrt{\sigma} P_{u r}^{u r}\right)=\int d^{D-2} x T s
$$

Hence the variation of the surface Hamiltonian leads to:

$$
\delta H_{\text {sur }}=-\frac{1}{4 \pi} \int d^{D-2} x\left[\delta\left(\sqrt{\mu} Q_{u r}^{u r}\right) \alpha+\sqrt{\mu} Q_{u r}^{u r} \delta \alpha\right]
$$

As well as we have the following expression from Eq. (62),

$$
\delta H_{\text {sur }}^{(1)}=\frac{1}{16 \pi} \int d^{D-2} x n_{c} U_{a}^{b d c} \delta \Gamma_{b d}^{a}=\frac{1}{4 \pi} \int d^{D-2} x \sqrt{\mu} Q_{u r}^{u r} \delta \alpha
$$

This along with Eqs. (81) and (62) leads to,

$$
\delta H_{\text {sur }}^{(2)}=\frac{1}{16 \pi} \int d^{D-1} x n_{c} \Gamma_{b d}^{a} \delta U_{a}^{b d c}=\frac{1}{4 \pi} \int d^{D-1} x \alpha \delta\left(\sqrt{\mu} Q_{u r}^{u r}\right)
$$

Then Eqs. (82) and (83) can also be interpreted in terms of entropy density $s=(m / 2) \sqrt{\mu} Q_{u r}^{u r}$ leading to:

$$
\begin{aligned}
\delta H_{\text {sur }}^{(1)} & =\int d^{D-2} x \frac{\sqrt{\mu} Q_{u r}^{u r}}{2} \delta\left(\frac{\alpha}{2 \pi}\right) \\
& =\frac{1}{m} \int d^{D-2} x s \delta T \\
\delta H_{\text {sur }}^{(2)} & =\int d^{D-2} x \frac{\alpha}{2 \pi} \delta\left(\frac{\sqrt{\mu} Q_{u r}^{u r}}{2}\right) \\
& =\frac{1}{m} \int d^{D-2} x T \delta s
\end{aligned}
$$

The above results hold for $m$ th order Lanczos-Lovelock Lagrangian, which can be generalized in a straight forward manner to a general Lanczos-Lovelock Lagrangian made of sum of individual Lanczos-Lovelock Lagrangians of different order.

For the sake of completeness, we mention that the same results can also be obtained for arbitrary variations if we impose a constraint on the background metric. This constraint is identical in general relativity and in Lanczos-Lovelock models and is given by $\partial_{u} \mu_{A B}=0$ and $\hat{D}_{M} \alpha=\partial_{u}\left(\beta_{M} / 2\right)$. This is evident from the components of curvature tensor presented in Eqs. (A12), (A13) and (A14). As all of them vanish, when we impose the condition $\partial_{u} \mu_{A B}=0$ condition, from Eqs. (74), (75), (76) and Eq. (79) we observe that only $Q_{u r}^{u r}$ term remains. This again leads to the standard expressions as in Eqs. (84) and (85).

\section{DESCRIBING GRAVITY IN TERMS OF CONJUGATE VARIABLES}

In the previous section we have shown how variations of the two variables introduced in Sec. III B are related to variations of temperature and entropy respectively. Having addressed the thermodynamic features for these variables we now study whether the gravitational dynamics can be described in terms of these variables. 


\section{A. Einstein-Hilbert Action with the new set of variables}

We start with the derivation of equations of motion in terms of the variables $f^{i j}$ and $N_{i j}^{k}$ used to describe Einstein-Hilbert action which was pointed out in [33]. We shall consider a Palatini-type variation in which we have taken the two conjugate variables, $f^{i j}$ and $N_{i j}^{k}$ as independent. For our purpose we shall define the Hamiltonian functional as

$$
\mathcal{H}_{g}=f^{a b}\left(N_{a d}^{c} N_{b c}^{d}-\frac{1}{3} N_{a c}^{c} N_{b d}^{d}\right) .
$$

Then the usual Hamilton's equations of motion obtained from the above Hamiltonian will be:

$$
\begin{aligned}
\partial_{c} f^{a b} & =\frac{\partial \mathcal{H}_{g}}{\partial N_{a b}^{c}} \\
& =f^{a d} N_{c d}^{b}+f^{b d} N_{c d}^{a}-\frac{1}{3} f^{a m} N_{d m}^{d} \delta_{c}^{b}-\frac{1}{3} f^{b m} N_{d m}^{d} \delta_{c}^{a} \\
\partial_{c} N_{a b}^{c} & =-\frac{\partial \mathcal{H}_{g}}{\partial f^{a b}} \\
& =-N_{a d}^{c} N_{b c}^{d}+\frac{1}{3} N_{a c}^{c} N_{b d}^{d}
\end{aligned}
$$

It is easy to show that these equations lead to (i) the correct relation between $f^{a b}$ and $N_{a b}^{c}$ and (ii) vacuum Einstein's equations $R_{a b}=0$. (For details, see Ref. [33]). (It is this result which actually justifies a posteriori our calling $\mathcal{H}_{g}$ the "Hamiltonian" and Eq. (88) the 'Hamilton's equations'; the structure is quite different from the corresponding ideas in standard ADM approach; see [30] for more details of this approach.)

We shall now try to generalize these ideas to Lanczos-Lovelock theories. As we have already noted in Sec. III B, the natural generalization of these two variables does not work in Lanczos-Lovelock models and we need to use the variable introduced in Eq. (32). It is therefore important to first verify what happens to general relativity when we use these variables. So we will first study the Einstein-Hilbert case before proceeding to Lanczos-Lovelock theory. For this purpose we shall start with the Einstein-Hilbert Lagrangian written as:

$$
\begin{aligned}
L & =\sqrt{-g} R=\sqrt{-g} \frac{1}{2}\left(g^{b d} \delta_{a}^{c}-g^{b c} \delta_{a}^{d}\right) R_{b c d}^{a} \\
& =\sqrt{-g}\left(g^{b d} \delta_{a}^{c}-g^{b c} \delta_{a}^{d}\right)\left(\partial_{c} \Gamma_{b d}^{a}-\Gamma_{d p}^{a} \Gamma_{b c}^{p}\right) \\
& \equiv U_{a}^{b c d}\left(\partial_{c} \Gamma_{b d}^{a}-\Gamma_{d p}^{a} \Gamma_{b c}^{p}\right)
\end{aligned}
$$

where we have defined the variable, $U_{a}^{b c d}=\sqrt{-g}\left(g^{b d} \delta_{a}^{c}-g^{b c} \delta_{a}^{d}\right)$ having all the symmetries of curvature tensor. Let us see what happens if we treat the variables $U_{a}^{b c d}$ and $\Gamma_{b c}^{a}$ as independent as befitting conjugate variables. The variation of $\Gamma_{b c}^{a}$ leads to:

$$
\begin{aligned}
\left.\delta L\right|_{U_{a} b c d} & =U_{a}^{b c d}\left(\partial_{c} \delta \Gamma_{b d}^{a}-\Gamma_{b c}^{p} \delta \Gamma_{d p}^{a}-\Gamma_{d p}^{a} \delta \Gamma_{b c}^{p}\right) \\
& =U_{a}^{b c d} \nabla_{c} \delta \Gamma_{b d}^{a}
\end{aligned}
$$

In order to get to the final expression we have used the fact that the Lagrangian is a scalar density and hence can be evaluated in a local inertial frame with $U_{a}^{b c d}$ and $\delta \Gamma_{b c}^{a}$ as tensors. (One can explicitly verify this result, even without using this trick.) Then we can rewrite Eq. (90) as:

$$
\left.\delta L\right|_{U_{a} b c d}=\nabla_{c}\left(U_{a}^{b c d} \delta \Gamma_{b d}^{a}\right)-\delta \Gamma_{b d}^{a} \nabla_{c} U_{a}^{b c d}
$$

When the above equation integrated over the spacetime volume to obtain the variation $\delta L$ of the action, the first term $\nabla_{c}\left(U_{a}{ }^{b c d} \delta \Gamma_{b d}^{a}\right)$, being a total divergence contributes only on the surface and hence can be dropped. Thus the condition $\delta L=0$ for arbitrary variations $\delta \Gamma_{b c}^{a}$ leads to:

$$
\nabla_{c} U_{a}^{b c d}=0
$$

From the definition of $U_{a}^{b c d}$ it is evident that Eq. (92) implies $\nabla_{c} g_{a b}=0$, standard result from Palatini variation in Einstein-Hilbert action [3] [This result can also be obtained using Eq. (A3)]. 
But we are led to a difficulty in this approach when we vary $U_{a}{ }^{b c d}$ because the variation of $U_{a}{ }^{b c d}$ leads to $R_{b c d}^{a}=0$, i.e. flat spacetime! It is not possible to get Einstein equation from arbitrary variations of $U_{a}^{b c d}$ since it has four indices, naturally leading to zero curvature tensor, equivalently flat spacetime. The reason for this disaster is simple. When we vary $U_{a}^{b c d}$ we are pretending that we are varying 20 independent components (because $U_{a}^{b c d}$ has the symmetries of curvature tensor); but we know that - since $U_{a}^{b c d}$ is completely determined by $g_{a b}$ - it really has only 10 independent components. So, in order to get correct equations of motion we need to restrict variations such that there are only 10 of them independent components in the variation $\delta U_{a}^{b c d}$. This is easy to achieve. Since an arbitrary symmetric second rank tensor $S_{q}^{p}$ has 10 independent components, we can easily construct such a constrained variation by considering a subclass of $\delta U_{a}^{b c d}$ which is determined by the variations $\delta S_{q}^{p}$ of an arbitrary second rank tensor. This leads us to consider variations of the form:

$$
\delta U_{p}{ }^{q r s}=\left(U_{m}^{q r s} \delta_{p}^{n}-\frac{1}{2} U_{p}^{q r s} \delta_{m}^{n}\right) \delta S_{n}^{m}
$$

(In fact, it turns out that the above variation can be slightly generalized by introducing a sixth rank tensor $A_{p m}^{q r s n}$ satisfying the criteria $A_{p m}^{q r s n} R_{q r s}^{p}=0$. We shall not consider these variations any more since they have no effect on the equation of motion.) With these restricted class of variations we arrive at:

$$
\begin{aligned}
\left.\delta L\right|_{\Gamma_{b c}^{a}} & =\frac{1}{2} R_{b c d}^{a} \delta U_{a}^{b c d} \\
& =\frac{1}{2} R^{p}{ }_{q r s}\left(U_{m}^{q r s} \delta_{p}^{n}-\frac{1}{2} \delta_{m}^{n} U_{p}{ }^{q r s}\right) \delta S_{n}^{m}
\end{aligned}
$$

Then for arbitrary variations of the symmetric tensor $S_{b}^{a}$, we get the equations of motion:

$$
R_{q r s}^{p}\left(U_{m}^{q r s} \delta_{p}^{n}-\frac{1}{2} \delta_{m}^{n} U_{p}^{q r s}\right)=0
$$

To prove the equivalence with Einstein equation we note that the following relations

$$
R^{p}{ }_{q r s} U_{m}^{q r s}=2 \sqrt{-g} R_{m}^{p} ; \quad R_{q r s}^{p} U_{p}^{q r s}=2 \sqrt{-g} R
$$

directly transform the equation of motion (95) to, $G_{a b}=0$, the source-free Einstein equation. (We have not included matter fields to our system about which we shall comment later.) In the next section we will show the validity of the above formalism for Lanczos-Lovelock gravity.

\section{B. Generalization to Lanczos-Lovelock gravity}

In the case of Lanczos-Lovelock models the appropriate Lagrangian to consider for our purpose is:

$$
L=U_{a}^{b c d}\left(\partial_{c} \Gamma_{b d}^{a}-\Gamma_{m d}^{a} \Gamma_{b c}^{m}\right)
$$

which, using Eq. (32), can be identified with the Lanczos-Lovelock Lagrangian. The variation of the above Lagrangian with respect to $\Gamma_{b c}^{a}$ leads to

$$
\nabla_{c} U_{a}^{b c d}=0
$$

as in the Einstein-Hilbert scenario (see Eq. (92)). This condition is equivalent to the criteria that in Lanczos-Lovelock gravity $\nabla_{c} P^{a b c d}=0$. This result is quiet remarkable, since the criterion that the field equation should be of second order in the dynamical variable gets into picture automatically from variation of the Lagrangian. In fact, this condition has another aspect to it. In general, when we study the metric formulation we treat the Lagrangian with $g_{a b}$ as the independent variable (with connections given in terms of the metric) while in the Palatini formulation, we treat both the metric and the connections as independent and their variation leads to the relation between them and the equation of motion. For an arbitrary Lagrangian the metric and Palatini variation do not coincide [51]. However if the condition $\nabla_{c}\left(\partial L / \partial R_{a b c d}\right)=0$ is satisfied then both the metric and Palatini formulations coincide. This is identical to the condition presented in Eq. (8) and it is interesting to see this condition emerging from a variation here. 
Next we need to vary the Lagrangian with respect to $U_{a}^{b c d}$. Arbitrary variation of $U_{a}^{b c d}$ treating all the 20 components independent will lead to trouble, just as in general relativity. Since the Lagrangian in Eq. (97) can equivalently be written as $L=(1 / 2) U_{a}^{b c d} R_{b c d}^{a}$, such that for arbitrary variation of $U_{a}^{b c d}$ we get $R_{b c d}^{a}=0$, i.e. flat spacetime solution - just as in general relativity. In order to get the field equation we need to again consider only a subclass of variations as we did in the Einstein-Hilbert scenario to derive the equations of motion in Eq. (95). Here again we need to assume that not all the independent components of $U_{a}^{b c d}$ are contributing to the variation but only 10 degrees of freedom, which can be encoded by a symmetric second rank part with arbitrary variation. This amounts to taking:

$$
\delta U_{p}^{q r s}=\left(U_{m}^{q r s} \delta_{p}^{n}-\frac{1}{2} U_{p}^{q r s} \delta_{m}^{n}\right) \delta S_{n}^{m}
$$

(Here also we can introduce an additional sixth rank tensor as we did after Eq. (93). However as far as the equation of motion is concerned it has no effect and thus will not be considered any more.) With these restricted class of variations the Lagrangian variation leads to:

$$
\left.\delta L\right|_{\Gamma_{b c}^{a}}=\left(m U_{a}^{p q r} \delta_{s}^{b}-\frac{1}{2} \delta_{a}^{b} U_{s}{ }^{p q r}\right) R_{p q r}^{s} \delta S_{b}^{a}
$$

where $\delta S_{b}^{a}$ is variation of an arbitrary symmetric second rank tensor and the factor $m$ comes from the fact that we are considering $m$ th order Lanczos-Lovelock Lagrangian. When the variation $\delta S_{b}^{a}$ is considered arbitrary the equation of motion turns out to be

$$
\left(m U_{a}^{p q r} \delta_{s}^{b}-\frac{1}{2} \delta_{a}^{b} U_{s}^{p q r}\right) R_{p q r}^{s}=0 .
$$

To show that the above equation of motion is indeed identical to the equation of motion in LanczosLovelock gravity we just use Eq. (32) to substitute for $U_{a}^{b c d}$ leading to:

$$
\begin{aligned}
0 & =m Q_{a}^{p q r} R_{p q r}^{b}-\frac{1}{2} \delta_{a}^{b} Q_{s}{ }^{p q r} R_{p q r}^{s} \\
& =\mathcal{R}_{b}^{a}-\frac{1}{2} \delta_{b}^{a} L
\end{aligned}
$$

which is the Lanczos-Lovelock equation of motion. Thus we observe that these two variables satisfy all the criteria that conjugate variables should.

The above result is derived for $m$ th order Lanczos-Lovelock Lagrangian and can be easily generalized to general Lanczos-Lovelock Lagrangian $L=\sum_{m} c_{m} L^{(m)}$. Then the above variation of Lanczos-Lovelock Lagrangian leads to the following expression:

$$
\begin{aligned}
\left.\delta(\sqrt{-g} L)\right|_{\Gamma_{b c}^{a}} & =\left.\sum_{m} c_{m} \delta\left(\sqrt{-g} L^{(m)}\right)\right|_{\Gamma_{b c}^{a}} \\
& =\left\{\left(\sum_{m} c_{m} m U_{a}{ }^{p q r}\right) \delta_{s}^{b}-\frac{1}{2} \delta_{a}^{b}\left(\sum_{m} c_{m} U_{s}{ }^{p q r}\right)\right\} R^{s}{ }_{p q r} \delta S_{b}^{a}
\end{aligned}
$$

For arbitrary variation of the symmetric tensor $S_{b}^{a}$ the equation of motion can be obtained as:

$$
\left\{\left(\sum_{m} c_{m} m U_{a}^{p q r}\right) \delta_{s}^{b}-\frac{1}{2} \delta_{a}^{b}\left(\sum_{m} c_{m} U_{s}^{p q r}\right)\right\} R_{p q r}^{s}=0
$$

Note that with the following relations

$$
\begin{array}{r}
\sum_{m} c_{m} m U_{a}{ }^{p q r}=\frac{\partial \sqrt{-g} L}{\partial R_{p q r}^{a}}=\sqrt{-g} P_{a}{ }^{p q r} \\
\sum_{m} c_{m} U_{s}^{p q r} R_{p q r}^{s}=\sqrt{-g} L
\end{array}
$$

the above Eq. (104) becomes equivalent to

$$
\mathcal{R}_{a}^{b}-\frac{1}{2} \delta_{a}^{b} L=0
$$


which is the Lanczos-Lovelock field equation.

In this approach, we arrive at the condition that needs to be imposed in order to get second order equation of motion, directly from a variational principle along with the field equation. The price we pay is the following: (a) We need to restrict the form of the variations, the physical meaning of which is unclear. (b) The inclusion of matter in this scheme is difficult. Usually, the energy momentum tensor comes from the variation of the matter Lagrangian with respect to the metric alone and since we have not included the metric in our formulation it is not clear how to include matter. These issues require further investigation.

\section{CONCLUDING REMARKS}

The link between the standard approach to gravity and the thermodynamical one is provided by the action principle of gravity. Previous studies have shown that these actions have several peculiar features and - under suitable conditions - a thermodynamical interpretation. This motivates us to look for geometrical variables in which the expression for action simplifies and which will have direct thermodynamical interpretation. More specifically, we want to discover geometrical variables, symbolically called $[q, p]$ such that $q \delta p$ and $p \delta q$ will correspond to $s \delta T$ and $T \delta s$ where $T$ is the horizon temperature and $s$ is the entropy density.

This goal was achieved for the Einstein-Hilbert action recently [33] by introducing canonically conjugate variables as $f^{a b}=\sqrt{-g} g^{a b}$ and the corresponding momenta $N_{a b}^{c}$. In terms of these variables, the surface term turns out to have the structure $-\partial(q p)$ and the their variations have direct thermodynamic interpretation.

It has been noticed in the past that virtually every result involving the thermodynamical interpretation gravity, which was valid for general relativity, could be generalized to Lanczos-Lovelock models. We have shown that this fact holds for the above result as well. We could introduce two suitable variables in the case of Lanczos-Lovelock models with the following properties: (a) These variables reduce to the ones used in general relativity in $D=4$ when the Lanczos-Lovelock model reduces to general relativity. (b) The variation of these quantities correspond to $s \delta T$ and $T \delta s$ where $s$ is now the correct Wald entropy density of the Lanczos-Lovelock model. This result holds rather trivially on any static (but not necessarily spherically symmetric or matter-free) horizon and - more importantly — on any arbitrary null surface acting as local Rindler horizon. Since local Rindler structures can be imposed on any event, this shows that, around any event, certain geometric variables can be attributed thermodynamical significance.

These variables, by themselves, seem interesting and deserves further study. For example, we found that they can be thought of as connections and conjugate momenta associated with connections in a formal sense. But to get sensible equations of motion by varying these quantities, we needed to restrict their variation in a manner which — while mathematically rigorous - is physically unclear. We thus find that while the thermodynamic significance of these variables are clear and direct, the dynamical significance requires further work to establish.

The analysis once again confirms that the thermodynamic interpretation goes far deeper than general relativity and is definitely telling us something nontrivial about the structure of the spacetime. We note that the nature of Wald entropy density in Lanczos-Lovelock models is far more complicated than a simple constant $(1 / 4)$ in general relativity ; yet, everything works out exactly as expected. The action principle somehow encodes the information about horizon thermodynamics, which is a key result in emergent gravity paradigm.

\section{Acknowledgement}

Research of T.P is partially supported by J.C. Bose research grant of DST, Govt. of India. Research of S.C is funded by a SPM Fellowship from CSIR, Govt. of India. S.C also likes to thank Krishnamohan Parattu, Suprit Singh, Bibhas Ranjan Majhi and Kinjalk Lochan for helpful discussions. We thank Naresh Dadhich for useful comments.

\section{Appendix A: Derivation of Various Identities used in Text}

We will consider the $p \partial q$ and $q \partial p$ structure arising from the identification of $\tilde{f}^{a b}$ as coordinate and $\tilde{N}_{a b}^{c}$ as momentum in Lanczos-Lovelock gravity. For the calculation, the following identity will be used here 
and there:

$$
\begin{aligned}
0=\nabla_{c} Q_{a b}^{c d} & =\partial_{c} Q_{a b}^{c d}+\Gamma_{c k}^{c} Q_{a b}^{k d} \\
& -\Gamma_{c a}^{k} Q_{k b}^{c d}-\Gamma_{c b}^{k} Q_{a k}^{c d}+\Gamma_{c k}^{d} Q_{a b}^{c k}
\end{aligned}
$$

However $Q_{a b}^{c d}$ being antisymmetric in (c,d) while $\Gamma_{a b}^{c}$ being symmetric in $(\mathrm{a}, \mathrm{b})$ the last term in the above expansion vanishes. Thus ordinary derivative of the quantity $Q_{a b}^{c d}$ has the following expression

$$
\partial_{c} Q_{a b}^{c d}=-\Gamma_{c k}^{c} Q_{a b}^{k d}+\Gamma_{c a}^{k} Q_{k b}^{c d}+\Gamma_{c b}^{k} Q_{a k}^{c d}
$$

Note that we can include $\sqrt{-g}$ in the above expression leading to:

$$
\partial_{c}\left(\sqrt{-g} Q_{a}^{b c d}\right)=\left(\sqrt{-g} Q_{p}^{b c d}\right) \Gamma_{a c}^{p}-\left(\sqrt{-g} Q_{a}^{p c d}\right) \Gamma_{c p}^{b}
$$

Thus we get the following expression from Eq. (A2):

$$
\begin{aligned}
\partial_{c} \tilde{N}_{a b}^{c} & =\partial_{c}\left[Q_{b p}^{c q} \Gamma_{a q}^{p}+Q_{a p}^{c q} \Gamma_{b q}^{p}\right] \\
& =\left(\partial_{c} Q_{b p}^{c q}\right) \Gamma_{a q}^{p}+Q_{b p}^{c q} \partial_{c} \Gamma_{a q}^{p}+\left(\partial_{c} Q_{a p}^{c q}\right) \Gamma_{b q}^{p}+Q_{a p}^{c q} \partial_{c} \Gamma_{b q}^{p} \\
& =Q_{k p}^{c q} \Gamma_{c b}^{k} \Gamma_{a q}^{p}+Q_{b k}^{c q} \Gamma_{c p}^{k} \Gamma_{a q}^{p}-Q_{b p}^{k q} \Gamma_{a q}^{p} \Gamma_{c k}^{c}+Q_{k p}^{c q} \Gamma_{c a}^{k} \Gamma_{b q}^{p} \\
& +Q_{a k}^{c q} \Gamma_{c p}^{k} \Gamma_{b q}^{p}-Q_{a p}^{k q} \Gamma_{c k}^{c} \Gamma_{b q}^{p}+Q_{b p}^{c q} \partial_{c} \Gamma_{a q}^{p}+Q_{a p}^{c q} \partial_{c} \Gamma_{b q}^{p}
\end{aligned}
$$

where in order to arrive at the last equality Eq. (A2) has been used. Now contracting the above expression with $\tilde{f}^{a b}$ we readily obtain

$$
\begin{aligned}
\tilde{f}^{a b} \partial_{c} \tilde{N}_{a b}^{c} & =\sqrt{-g} g^{a b}\left[2 Q_{a p}^{c q} \partial_{c} \Gamma_{b q}^{p}+2 Q_{k p}^{c q} \Gamma_{c a}^{k} \Gamma_{b q}^{p}+2 Q_{a k}^{c q} \Gamma_{c p}^{k} \Gamma_{b q}^{p}-2 Q_{a p}^{k q} \Gamma_{c k}^{c} \Gamma_{b q}^{p}\right] \\
& =-2 \sqrt{-g} Q_{p}^{b c q}\left(\partial_{c} \Gamma_{b q}^{p}+\Gamma_{c k}^{p} \Gamma_{b q}^{k}\right) \\
& +2 \sqrt{-g} Q_{p}^{b k q} \Gamma_{c k}^{c} \Gamma_{b q}^{p}+2 \sqrt{-g} g^{a b} Q_{k p}^{c q} \Gamma_{c a}^{k} \Gamma_{b q}^{p} \\
& =-\sqrt{-g} Q_{p}^{b q c} R_{b q c}^{p}+2 \sqrt{-g} Q_{p}^{b k q} \Gamma_{c k}^{c} \Gamma_{b q}^{p}+2 \sqrt{-g} g^{a b} Q_{k p}^{c q} \Gamma_{c a}^{k} \Gamma_{b q}^{p}
\end{aligned}
$$

Note that in the Einstein-Hilbert limit the last two terms adds up to yield $-\sqrt{-g} L_{\text {quad }}$. Then consider the other combination which can be expressed as

$$
\begin{aligned}
\tilde{N}_{a b}^{c} \partial_{c} \tilde{f}^{a b} & =\left(Q_{a p}^{c q} \Gamma_{q b}^{p}+Q_{b p}^{c q} \Gamma_{q a}^{p}\right) \partial_{c}\left(\sqrt{-g} g^{a b}\right) \\
& =\sqrt{-g}\left(Q_{a p}^{c q} \Gamma_{q b}^{p}+Q_{b p}^{c q} \Gamma_{q a}^{p}\right)\left(\partial_{c} g^{a b}+g^{a b} \Gamma_{c p}^{p}\right) \\
& =2 \sqrt{-g} Q_{a p}^{c q} \Gamma_{q b}^{p} \partial_{c} g^{a b}+2 \sqrt{-g} Q_{p}^{b q c} \Gamma_{q b}^{p} \Gamma_{c m}^{m} \\
& =2 \sqrt{-g} Q_{p}^{b c q} \Gamma_{b c}^{l} \Gamma_{q l}^{p}+2 \sqrt{-g} Q_{p}^{b q c} \Gamma_{q b}^{p} \Gamma_{c m}^{m}-2 \sqrt{-g} g^{b m} Q_{a p}^{c q} \Gamma_{q b}^{p} \Gamma_{c m}^{a} \\
& =\sqrt{-g} L_{q u a d}+2 \sqrt{-g} Q_{p}^{b q c} \Gamma_{q b}^{p} \Gamma_{c m}^{m}-2 \sqrt{-g} g^{b m} Q_{a p}^{c q} \Gamma_{q b}^{p} \Gamma_{c m}^{a} .
\end{aligned}
$$

In the Einstein-Hilbert limit the above term leads to $2 \sqrt{-g} L_{\text {quad }}$. Next we will derive similar relations which actually behaves as conjugate variables, with the identification, $p \equiv 2 \sqrt{-g} Q_{a}^{b c d}$ and $q \equiv \Gamma_{b c}^{a}$. Then the respective $p \partial q$ and $q \partial p$ expressions are given in the following results:

$$
\begin{aligned}
2 \sqrt{-g} Q_{e}^{b d c} \partial_{c} \Gamma_{b d}^{e} & =\sqrt{-g} Q_{e}^{b d c}\left(\partial_{c} \Gamma_{b d}^{e}-\partial_{d} \Gamma_{b c}^{e}\right) \\
& =\sqrt{-g} Q_{e}^{b d c} R_{b c d}^{e}-2 \sqrt{-g} Q_{e}^{b d c} \Gamma_{m c}^{e} \Gamma_{b d}^{m} \\
& =-\sqrt{-g} Q_{e}^{a b c} R_{a b c}^{e}-\sqrt{-g} L_{q u a d}
\end{aligned}
$$

and

$$
\begin{aligned}
\Gamma_{b e}^{d} \partial_{c}\left(2 \sqrt{-g} Q_{d}^{b e c}\right) & =2 \sqrt{-g} \Gamma_{b e}^{d} \partial_{c} Q_{d}^{b e c}+2 \Gamma_{b e}^{d} Q_{d}^{b e c} \partial_{c} \sqrt{-g} \\
& =2 \sqrt{-g} \Gamma_{b e}^{d}\left(\Gamma_{c d}^{a} Q_{a}^{b e c}-\Gamma_{c a}^{b} Q_{d}^{a e c}-\Gamma_{c a}^{c} Q_{d}^{b e a}\right)+2 \Gamma_{b e}^{d} Q_{d}^{b e c} \partial_{c} \sqrt{-g} \\
& =2 \sqrt{-g} L_{\text {quad }}
\end{aligned}
$$


Now we will show one derivative used in the text for Lanczos-Lovelock Lagrangian:

$$
\begin{aligned}
\frac{\partial(\sqrt{-g} L)}{\partial\left(\partial_{l} \Gamma_{v w}^{u}\right)} & =m \sqrt{-g} \delta_{c d c_{2} d_{2} \ldots c_{m} d_{m}}^{a b a_{2} b_{2} \ldots a_{m} b_{m}} \frac{\partial R_{a b}^{c d}}{\partial\left(\partial_{l} \Gamma_{v w}^{u}\right)} R_{a_{2} b_{2}}^{c_{2} d_{2}} \ldots R_{a_{m} b_{m}}^{c_{m} d_{m}} \\
& =m \sqrt{-g} \delta_{c d c_{2} d_{2} \ldots c_{m} d_{m}}^{a b a_{2} b_{2} \ldots a_{m} b_{m}}\left[g^{d p} \delta_{u}^{c} \delta_{p}^{v}\left(\delta_{a}^{l} \delta_{b}^{w}-\delta_{b}^{l} \delta_{a}^{w}\right)\right] R_{a_{2} b_{2}}^{c_{2} d_{2}} \ldots R_{a_{m} b_{m}}^{c_{m} d_{m}} \\
& =2 m \sqrt{-g} g^{d v} Q_{u d}^{l w}=m U_{u}^{v l w}
\end{aligned}
$$

The next thing to consider are the connections in the general static metric we are considering. There the connections are given by

$$
\begin{aligned}
\Gamma_{n n}^{n} & =\Gamma_{t n}^{n}=\Gamma_{n A}^{n}=\Gamma_{t A}^{n}=0 \\
\Gamma_{t t}^{n} & =N \partial_{n} N ; \quad \Gamma_{A B}^{n}=-\frac{1}{2} \partial_{n} \sigma_{A B} \\
\Gamma_{n n}^{t} & =\Gamma_{n A}^{t}=0 \\
\Gamma_{n t}^{t} & =\frac{\partial_{n} N}{N} ; \quad \Gamma_{t t}^{t}=\frac{\partial_{t} N}{N} \\
\Gamma_{t A}^{t} & =\frac{\partial_{A} N}{N} ; \quad \Gamma_{A B}^{t}=\frac{\partial_{t} \sigma_{A B}}{2 N^{2}} \\
\Gamma_{n n}^{A} & =\Gamma_{n t}^{A}=0 \\
\Gamma_{n B}^{A} & =\frac{1}{2} \sigma^{A C}\left(\partial_{n} \sigma_{B C}\right) ; \quad \Gamma_{t t}^{A}=N \sigma^{A B} \partial_{B} N \\
\Gamma_{t B}^{A} & =\frac{1}{2} \sigma^{A C}\left(\partial_{t} \sigma_{B C}\right) ; \quad \Gamma_{B C}^{A}=\frac{1}{2} \sigma^{A D}\left(-\partial_{D} \sigma_{B C}+\partial_{B} \sigma_{C D}+\partial_{C} \sigma_{B D}\right)
\end{aligned}
$$

Also we list below all the connections in GNC coordinate system that will remain nonzero in the null surface limit:

$$
\begin{aligned}
\Gamma_{u u}^{u} & =\alpha ; \quad \Gamma_{u A}^{u}=\beta_{A} / 2 ; \quad \Gamma_{A B}^{u}=-\partial_{r} \mu_{A B} / 2 \\
\Gamma_{u r}^{r} & =-\alpha ; \quad \Gamma_{r A}^{r}=-\beta_{A} / 2 ; \quad \Gamma_{A B}^{r}=-\partial_{u} \mu_{A B} / 2 \\
\Gamma_{B C}^{A} & =\hat{\Gamma}_{B C}^{A} ; \quad \Gamma_{B u}^{A}=\mu^{C A} \partial_{u} \mu_{B C} / 2 ; \\
\Gamma_{B r}^{A} & =\mu^{C A} \partial_{r} \mu_{B C} / 2 ; \quad \Gamma_{u r}^{A}=-\beta^{A} / 2
\end{aligned}
$$

Now we will present curvature tensor components in GNC coordinates, which are relevant for the calculations in the main text

$$
\begin{aligned}
R_{P u B}^{C} & =\partial_{u} \Gamma_{P B}^{C}-\partial_{B} \Gamma_{P u}^{C}+\Gamma_{m u}^{C} \Gamma_{P B}^{m}-\Gamma_{m B}^{C} \Gamma_{P u}^{m} \\
& =\partial_{u}\left(\frac{1}{2} \bar{\beta}^{C} \partial_{r} \mu_{P B}+\hat{\Gamma}_{P B}^{C}\right)-\partial_{B}\left(\frac{1}{2} \bar{\beta}^{C} \partial_{r} \bar{\beta}_{P}+\frac{1}{2} \mu^{C A} \partial_{u} \mu_{P A}+\frac{1}{2} \mu^{C A}\left(\hat{D}_{P} \bar{\beta}_{A}-\hat{D}_{A} \bar{\beta}_{P}\right)\right) \\
& +\left(\frac{1}{2} \bar{\beta}^{C} \partial_{r} \bar{\beta}_{A}+\frac{1}{2} \mu^{C B} \partial_{u} \mu_{B A}+\frac{1}{2} \mu^{C B}\left(\hat{D}_{A} \bar{\beta}_{B}-\hat{D}_{B} \bar{\beta}_{A}\right)\right)\left(\frac{1}{2} \bar{\beta}^{A} \partial_{r} \mu_{B P}+\hat{\Gamma}_{B P}^{A}\right) \\
& -\left(\frac{1}{2} \bar{\beta}^{C} \partial_{r} \bar{\alpha}-\frac{1}{2} \mu^{C A} \hat{D}_{A} \bar{\alpha}+\mu^{A C} \partial_{u} \bar{\beta}_{A}\right)\left(\frac{1}{2} \partial_{r} \mu_{A B}\right) \\
& -\left(\frac{1}{2} \mu^{C A} \partial_{r} \bar{\beta}_{A}\right)\left(\frac{1}{2}\left\{\partial_{u} \mu_{P B}+\left(\bar{\beta}^{C} \bar{\beta}_{C}-\bar{\alpha}\right) \partial_{r} \mu_{P B}\right\}+\frac{1}{2}\left(\hat{D}_{P} \bar{\beta}_{B}+\hat{D}_{B} \bar{\beta}_{P}\right)\right) \\
& -\left(\frac{1}{2} \bar{\beta}^{C} \partial_{r} \mu_{B A}+\hat{\Gamma}_{A B}^{C}\right)\left(\frac{1}{2} \bar{\beta}^{A} \partial_{r} \bar{\beta}_{P}+\frac{1}{2} \mu^{C A} \partial_{u} \mu_{P C}+\frac{1}{2} \mu^{C A}\left(\hat{D}_{P} \bar{\beta}_{C}-\hat{D}_{C} \bar{\beta}_{P}\right)\right) \\
& +\left(\frac{1}{2} \bar{\beta}^{C} \partial_{r} \bar{\beta}_{B}+\frac{1}{2} \mu^{C A} \partial_{u} \mu_{B A}+\frac{1}{2} \mu^{C A}\left(\hat{D}_{B} \bar{\beta}_{A}-\hat{D}_{A} \bar{\beta}_{B}\right)\right)\left(\frac{1}{2} \partial_{r} \bar{\beta}_{P}\right) \\
& +\left(\frac{1}{2} \mu^{C A} \partial_{r} \mu_{B A}\right)\left(\frac{1}{2}\left(\bar{\beta}^{C} \bar{\beta}_{C}-\bar{\alpha}\right) \partial_{r} \bar{\beta}_{P}+\frac{1}{2} \hat{D}_{P} \bar{\alpha}-\frac{1}{2} \bar{\beta}^{B}\left(\partial_{u} \mu_{P B}+\hat{D}_{P} \bar{\beta}_{B}-\hat{D}_{B} \bar{\beta}_{P}\right)\right) \\
& =\partial_{u} \hat{\Gamma}_{P B}^{C}-\frac{1}{2} \mu^{C M} \partial_{B} \partial_{u} \mu_{M P}-\frac{1}{2} \partial_{B} \mu^{C M} \partial_{u} \mu_{M P}+\frac{1}{2} \mu^{C M} \partial_{u} \mu_{M A} \hat{\Gamma}_{P B}^{A} \\
& -\frac{1}{2} \partial_{u} \mu_{P B} \Gamma_{r u}^{C}-\frac{1}{2} \mu^{C M} \partial_{u} \mu_{M B} \Gamma_{P u}^{u}-\frac{1}{2} \mu^{A C} \partial_{u} \mu_{C P} \hat{\Gamma}_{A B}^{C}
\end{aligned}
$$




$$
\begin{aligned}
R_{A B u}^{r} & =\partial_{B} \Gamma_{A u}^{r}-\partial_{u} \Gamma_{A B}^{r}+\Gamma_{B m}^{r} \Gamma_{A u}^{m}-\Gamma_{u m}^{r} \Gamma_{B A}^{m} \\
& =\partial_{B}\left[-\frac{1}{2}\left(\bar{\beta}^{C} \bar{\beta}_{C}-\bar{\alpha}\right) \partial_{r} \bar{\beta}_{A}+\frac{1}{2} \hat{D}_{A} \bar{\alpha}-\frac{1}{2} \bar{\beta}^{B}\left(\partial_{u} \mu_{A B}+\hat{D}_{A} \bar{\beta}_{B}-\hat{D}_{B} \bar{\beta}_{A}\right)\right] \\
& -\partial_{u}\left[-\frac{1}{2}\left\{\partial_{u} \mu_{A B}+\left(\bar{\beta}^{C} \bar{\beta}_{C}-\bar{\alpha}\right) \partial_{r} \mu_{A B}\right\}+\frac{1}{2}\left(\hat{D}_{A} \bar{\beta}_{B}+\hat{D}_{B} \bar{\beta}_{A}\right)\right] \\
& +\left(\left[\frac{1}{2}\left(\partial_{r} \bar{\beta}_{B}-\bar{\beta}^{C} \partial_{r} \mu_{C B}\right)\right]\left[-\frac{1}{2}\left(\bar{\beta}^{C} \bar{\beta}_{C}-\bar{\alpha}\right) \partial_{r} \bar{\beta}_{A}+\frac{1}{2} \hat{D}_{A} \bar{\alpha}-\frac{1}{2} \bar{\beta}^{B}\left(\partial_{u} \mu_{A B}+\hat{D}_{A} \bar{\beta}_{B}-\hat{D}_{B} \bar{\beta}_{A}\right)\right]\right. \\
& +\left[-\frac{1}{2}\left(\bar{\beta}^{C} \bar{\beta}_{C}-\bar{\alpha}\right) \partial_{r} \bar{\beta}_{A}+\frac{1}{2} \hat{D}_{A} \bar{\alpha}-\frac{1}{2} \bar{\beta}^{B}\left(\partial_{u} \mu_{A B}+\hat{D}_{A} \bar{\beta}_{B}-\hat{D}_{B} \bar{\beta}_{A}\right)\right]\left[-\frac{1}{2} \partial_{r} \bar{\beta}_{A}\right] \\
& +\left[-\frac{1}{2}\left\{\partial_{u} \mu_{B C}+\left(\bar{\beta}^{M} \bar{\beta}_{M}-\bar{\alpha}\right) \partial_{r} \mu_{B C}\right\}+\frac{1}{2}\left(\hat{D}_{C} \bar{\beta}_{B}+\hat{D}_{B} \bar{\beta}_{C}\right)\right] \\
& \left.\times\left[\frac{1}{2} \bar{\beta}^{C} \partial_{r} \bar{\beta}_{A}+\frac{1}{2} \mu^{C B} \partial_{u} \mu_{B A}+\frac{1}{2} \mu^{C B}\left(\hat{D}_{B} \bar{\beta}_{A}-\hat{D}_{A} \bar{\beta}_{B}\right)\right]-(B \leftrightarrow u)\right) \\
& =\frac{1}{2} \partial_{u}^{2} \mu_{A B}-\frac{1}{4} \partial_{u} \mu_{B C} \mu^{C M} \partial_{u} \mu_{M A}+\frac{1}{2} \alpha \partial_{u} \mu_{A B}
\end{aligned}
$$

and

$$
\begin{aligned}
R_{P Q R}^{r} & =\partial_{Q} \Gamma_{P R}^{r}-\partial_{R} \Gamma_{P Q}^{r}+\Gamma_{m Q}^{r} \Gamma_{P R}^{m}-\Gamma_{m R}^{r} \Gamma_{P Q}^{m} \\
& =-\partial_{Q}\left(\frac{1}{2}\left\{\partial_{u} \mu_{P R}+\left(\bar{\beta}^{C} \bar{\beta}_{C}-\bar{\alpha}\right) \partial_{r} \mu_{P R}\right\}+\frac{1}{2}\left(\hat{D}_{P} \bar{\beta}_{R}+\hat{D}_{R} \bar{\beta}_{P}\right)\right) \\
& +\left(\frac{1}{2}\left(\bar{\beta}^{C} \bar{\beta}_{C}-\bar{\alpha}\right) \partial_{r} \bar{\beta}_{Q}+\frac{1}{2} \hat{D}_{Q} \bar{\alpha}-\frac{1}{2} \bar{\beta}^{B}\left(\partial_{u} \mu_{Q B}+\hat{D}_{Q} \bar{\beta}_{B}-\hat{D}_{B} \bar{\beta}_{Q}\right)\right)\left(\frac{1}{2} \partial_{r} \mu_{P R}\right) \\
& -\left(\frac{1}{2}\left(\partial_{r} \bar{\beta}_{Q}-\bar{\beta}^{C} \partial_{r} \mu_{C Q}\right)\right)\left(\frac{1}{2}\left\{\partial_{u} \mu_{P R}+\left(\bar{\beta}^{C} \bar{\beta}_{C}-\bar{\alpha}\right) \partial_{r} \mu_{P R}\right\}+\frac{1}{2}\left(\hat{D}_{P} \bar{\beta}_{R}+\hat{D}_{R} \bar{\beta}_{P}\right)\right) \\
& -\left(\frac{1}{2}\left\{\partial_{u} \mu_{A Q}+\left(\bar{\beta}^{C} \bar{\beta}_{C}-\bar{\alpha}\right) \partial_{r} \mu_{A Q}\right\}+\frac{1}{2}\left(\hat{D}_{A} \bar{\beta}_{Q}+\hat{D}_{Q} \bar{\beta}_{A}\right)\right)\left(\frac{1}{2} \bar{\beta}^{A} \partial_{r} \mu_{P R}+\hat{\Gamma}_{P R}^{A}\right)-(Q \leftrightarrow R) \\
& =-\frac{1}{2} \partial_{Q} \partial_{u} \mu_{P R}+\frac{1}{2} \partial_{R} \partial_{u} \mu_{P Q}+\frac{1}{4} \beta_{Q} \partial_{u} \mu_{P R}-\frac{1}{4} \beta_{R} \partial_{u} \mu_{P Q} \\
& -\frac{1}{2} \partial_{u} \mu_{A Q} \hat{\Gamma}_{P R}^{A}+\frac{1}{2} \partial_{u} \mu_{A R} \hat{\Gamma}_{P Q}^{A}
\end{aligned}
$$

\section{Appendix B: Identities Regarding Lie Variation of $P^{a b c d}$}

In this section we shall derive some identities related to Lie variation of the entropy tensor, $P^{a b c d}$. For that purpose we first consider Lie variation of the Lagrangian treated as a scalar function of the metric $g_{a b}$ and $R_{a b c d}$ leading to

$$
£_{\xi} L\left(g_{a b}, R_{i j k l}\right)=\xi^{m} \nabla_{m} L\left(g_{a b}, R_{i j k l}\right)=\frac{\partial L}{\partial g_{a b}} \xi^{m} \nabla_{m} g_{a b}+\frac{\partial L}{\partial R_{i j k l}} \xi^{m} \nabla_{m} R_{i j k l}=P^{i j k l} \xi^{m} \nabla_{m} R_{i j k l}
$$

where we have used the fact that covariant derivative of metric tensor vanishes. Then for the Lagrangian which is homogeneous function of degree $m$ we get

$$
£_{\xi} L=\xi^{m} \nabla_{m}\left(\frac{1}{m} P^{i j k l} R_{i j k l}\right)
$$

Then using Eq. (B1) we readily obtain

$$
R_{a b c d} \xi^{m} \nabla_{m} P^{a b c d}=(m-1) P^{a b c d} \xi^{m} \nabla_{m} R_{a b c d}
$$

We also have the following relation:

$$
\begin{aligned}
P^{i j k l} £_{\xi} R_{i j k l} & =P^{i j k l}\left(\xi^{m} \nabla_{m} R_{i j k l}+R_{a j k l} \nabla_{i} \xi^{a}+R_{i a k l} \nabla_{j} \xi^{a}+R_{i j a l} \nabla_{k} \xi^{a}+R_{i j k a} \nabla_{l} \xi^{a}\right) \\
& =P^{i j k l} \xi^{m} \nabla_{m} R_{i j k l}+4 \nabla_{i} \xi_{m} R^{i}{ }_{j k l} P^{m j k l} \\
& =P^{i j k l} \xi^{m} \nabla_{m} R_{i j k l}+4 \nabla_{i} \xi_{m} \mathcal{R}^{i m}
\end{aligned}
$$


Again we can also write, $m £_{\xi} L=P^{i j k l} £_{\xi} R_{i j k l}+R_{i j k l} £_{\xi} P^{i j k l}$. Then we obtain:

$$
\begin{aligned}
R_{i j k l} £_{\xi} P^{i j k l} & =m £_{\xi} L-P^{i j k l} £_{\xi} R_{i j k l} \\
& =m £_{\xi} L-P^{i j k l} \xi^{m} \nabla_{m} R_{i j k l}-4 \nabla_{i} \xi_{m} \mathcal{R}^{i m} \\
& =(m-1) P^{a b c d} \xi^{m} \nabla_{m} R_{a b c d}-4 \nabla_{i} \xi_{m} \mathcal{R}^{i m}
\end{aligned}
$$

This equation can also be casted in a different form as

$$
R_{a b c d}\left(£_{\xi} P^{a b c d}-\xi^{m} \nabla_{m} P^{a b c d}\right)=-4 \nabla_{i} \xi_{m} \mathcal{R}^{i m}
$$

Now we can rewrite the metric as a function of $g_{a b}$ and $R_{b c d}^{a}$, in which case the Lie variation leads to

$$
£_{\xi} L\left(g_{i j}, R_{b c d}^{a}\right)=P_{i}{ }^{j k l} \xi^{m} \nabla_{m} R_{j k l}^{i}
$$

With the Lagrangian as homogeneous function of curvature tensor to mth order leads to

$$
R_{b c d}^{a} £_{\xi} P_{a}^{b c d}=(m-1) P_{i}{ }^{j k l} \xi^{m} \nabla_{m} R_{j k l}^{i}
$$

Then we arrive at the following identity

$$
P^{a b c d} £_{\xi}\left(g_{a m} R_{b c d}^{m}\right)=P_{a}^{b c d} \xi^{m} \nabla_{m} R_{b c d}^{a}+4 \nabla_{i} \xi_{m} \mathcal{R}^{i m}
$$

or

$$
\begin{aligned}
P_{a}^{b c d} £_{\xi} R_{b c d}^{a} & =P_{a}^{b c d} \xi^{m} \nabla_{m} R_{b c d}^{a}+4 \nabla_{i} \xi_{m} \mathcal{R}^{i m}-\mathcal{R}^{a m} \mathcal{L}_{\xi} g_{a m} \\
& =P_{a}^{b c d} \xi^{m} \nabla_{m} R_{b c d}^{a}+2 \nabla_{i} \xi_{m} \mathcal{R}^{i m}
\end{aligned}
$$

This leads to the following relation:

$$
R_{b c d}^{a}\left(£_{\xi} P_{a}^{b c d}-\xi^{m} \nabla_{m} P_{a}^{b c d}\right)=-2 \nabla_{i} \xi_{m} \mathcal{R}^{i m}
$$

If we proceed along the same lines we readily obtain another such relation given as:

$$
R_{k l}^{i j}\left(£_{\xi} P_{i j}^{k l}-\xi^{m} \nabla_{m} P_{i j}^{k l}\right)=0
$$

These relations illustrate the Lie variation of $P^{a b c d}$ when contracted with the curvature tensor.

[1] C. Lanczos, Z.Phys. 73, 147 (1932);

C. Lanczos, Ann. of Math. 39, 842 (1938).

[2] D. Lovelock, J. Math. Phys. 12, 498 (1971).

[3] T. Padmanabhan, Gravitation: Foundation and Frontiers, Cambridge University Press, Cambridge, UK (2010).

[4] T. Padmanabhan and D. Kothawala, Phys. Rept. 531, 115 (2013).

[5] J.D. Bekenstein, Phys. Rev. D 7, 2333 (1973).

[6] J.D. Bekenstein, Phys. Rev. D 9, 3292 (1974).

[7] S. Hawking, Commun. Math. Phys. 43, 199 (1975).

[8] P. C. W. Davies, S. A. Fulling, and W. G. Unruh, Phys. Rev. D 13, 2720 (1976).

[9] W. G. Unruh, Phys. Rev. D 14, 870 (1976).

[10] G. Gibbons and S.W. Hawking, Phys. Rev. D 15, 2752 (1977).

[11] T. Padmanabhan, Phys. Rept. 406, 49 (2005) arXiv:0311036.

[12] T. Padmanabhan, Rept. Prog. Phys. 73, 046901 (2010) arXiv:0911.5004.

[13] R.M. Wald, Liv. Rev. Relt. 4, 6 (2001) arXiv:9912119.

[14] T. Padmanabhan, Class. Quant. Grav. 19, 5837 (2002) arXiv:0204019.

[15] R.G. Cai and S.P. Kim, J. High Energy Phys. 0502, 050 (2005) arXiv:hep-th/0501055.

[16] A. Paranjape, S. Sarkar and T. Padmanabhan, Phys. Rev. D 74, 104015 (2006) arXiv:hep-th/0607240.

[17] M. Akbar and R.G. Cai, Phys. Lett. B 635, 7 (2006) arXiv:hep-th/0602156.

[18] T. Padmanabhan, AIP Conference Proceedings 861, 858 (2006) arXiv:astro-ph/0603114.

[19] D. Kothawala and T. Padmanabhan, Phys. Rev. D 79, 104020 (2009) arXiv:0904.0215.

[20] A. Mukhopadhyay and T. Padmanabhan, Phys. Rev. D 74, 124023 (2006) arXiv:hep-th/0608120.

[21] S. Kolekar and T. Padmanabhan, Phys. Rev. D 82, 024036 (2010) arXiv:1005.0619. 
[22] S. Kolekar, D. Kothawala and T. Padmanabhan, Phys. Rev. D 85, 064031 (2012) arXiv:1111.0973.

[23] T. Padmanabhan and A. Paranjape, Phys. Rev. D 75, 064004 (2007) arXiv:0701003.

[24] T. Padmanabhan, Gen. Relt. Grav. 40, 529 (2008) arXiv:0705.2533.

[25] T. Padmanabhan, Braz. J. Phys. 35, 362 (2005) arXiv:0412068.

[26] T. Padmanabhan, Mod. Phys. Lett. A25, 1129 (2010) arXiv:0912.3165.

[27] S. Kolekar and T. Padmanabhan, Phys. Rev. D 85, 024004 (2012) arXiv:1109.5353.

[28] T. Padmanabhan, Phys. Rev. D 83, 044048 (2011) arXiv:1012.0119.

[29] T. Damour, Surface Effects in Black Hole Physics, Proceedings of the second Marcel Grossmann Meeting on General Relativity (1982).

[30] T. Padmanabhan, Gen. Rel. Grav 46, 1673 (2014) arXiv:1312.3253.

[31] S. Chakraborty and T. Padmanabhan Phys. Rev. D 90, 124017 (2014) arXiv:1408.4679.

[32] T. Padmanabhan, Phys. Rev. D 81, 124040 (2010) arXiv:1003.5665.

[33] K. Parattu, B.R. Majhi and T. Padmanabhan, Phys. Rev. D 87, 124011 (2013) arXiv:1303.1535.

[34] A. Yale and T. Padmanabhan, Gen. Relt. Grav. 43, 1549 (2011) arXiv:1008.5154; N. Kiriushcheva and S. Kuzmin, Mod. Phys. Lett. A21 (2006) arXiv:hep-th/0510260.

[35] T. Padmanabhan, Phys. Rev. D 84, 124041 (2011) arXiv:1109.3846.

[36] T. Padmanabhan, AIP Conf. Proc. 1241, 93 (2010) arXiv:0911.1403.

[37] B.R. Majhi and T. Padmanabhan, Phys. Rev. D 85, 084040 (2012) arXiv:1111.1809.

[38] R.M. Wald, Phys. Rev. D 48, R3427 (1993);

V. Iyer and R.M. Wald, Phys. Rev. D 50, 846 (1994);

R.M. Wald and A. Zoupas, Phys. Rev. D 61, 084027 (2000).

[39] A. Strominger and C. Vafa, Phys. Lett. B 379, 99 (1996).

[40] A. Ashtekar, J. Baez, A. Corichi and K. Krasnov, Phys. Rev. Lett. 80, 904 (1998); J.M. Garcia-Islas, Class. Quant. Grav. 25, 245001 (2008).

[41] L. Bombelli, R.K. Koul, J. Lee and R.D. Sorkin, Phys. Rev. D 34, 373 (1986).

[42] B.R. Majhi and T. Padmanabhan, Eur. Phys. J C 73, 2651 (2013) arXiv:1302.1206.

[43] A. Eddington, The Mathematical Theory of Relativity, Cambridge University Press, Cambridge, UK (1924); E. Schrodinger, Space-time Structure, Cambridge University Press, Cambridge, UK (1950).

[44] V. Moncrief and J. Isenberg, Commun. Math. Phys. 89, 387 (1983).

[45] E.M. Morales, On a Second Law of Black Hole Mechanics in a Higher Derivative Theory of Gravity Ph.D Thesis, Gottingen University.

[46] A. Medved, D. Martin and M. Visser, Class. Quant. Grav. 213111 (2004) arXiv:0402069.

[47] T. Padmanabhan, Gen. Relt. Grav. 44, 2681 (2012) arXiv:1205.5683.

[48] I. Racz and R.M. Wald, Class. Quanta. Grav. 13, 539 (1996) arXiv:9507055.

[49] B. Carter, Gen. Relt. Grav. 42, 653 (2010).

[50] K. Parattu, S. Chakraborty, B.R. Majhi and T. Padmanabhan (in preparation).

[51] Q. Exirifard and M.M. Sheikh-Jabbari, Phys. Lett. B 661, 158 (2008). 